\title{
Foxc1 and Foxc2 deletion causes abnormal lymphangiogenesis and correlates with ERK hyperactivation
}

\author{
Anees Fatima, ${ }^{1}$ Ying Wang, ${ }^{2}$ Yutaka Uchida, ${ }^{3}$ Pieter Norden, ${ }^{1}$ Ting Liu, ${ }^{1}$ Austin Culver, ${ }^{1}$ William H. Dietz, ${ }^{1}$ Ford Culver, ${ }^{1}$ \\ Meredith Millay, ${ }^{1}$ Yoh-suke Mukouyama, ${ }^{3}$ and Tsutomu Kume ${ }^{1}$ \\ ${ }^{1}$ Feinberg Cardiovascular Research Institute, Department of Medicine, Feinberg School of Medicine, Northwestern University, Chicago, Illinois, USA. ${ }^{2}$ Department of Biochemistry and Molecular Biology,

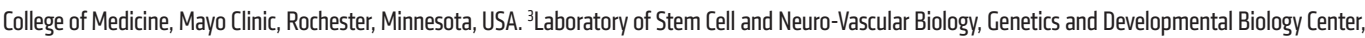 \\ National Heart, Lung, and Blood Institute, NIH, Bethesda, Maryland, USA.
}

\begin{abstract}
The lymphatic vasculature is essential for maintaining interstitial fluid homeostasis, and dysfunctional lymphangiogenesis contributes to various pathological processes, including inflammatory disease and tumor metastasis. Mutations in FOXC2 are dominantly associated with late-onset lymphedema; however, the precise role of FOXC2 and a closely related factor, FOXC1, in the lymphatic system remains largely unknown. Here we identified a molecular cascade by which FOXC1 and FOXC2 regulate ERK signaling in lymphatic vessel growth. In mice, lymphatic endothelial cell-specific (LEC-specific) deletion of Foxc1, Foxc2, or both resulted in increased LEC proliferation, enlarged lymphatic vessels, and abnormal lymphatic vessel morphogenesis. Compared with LECs from control animals, LECs from mice lacking both Foxc1 and Foxc2 exhibited aberrant expression of Ras regulators, and embryos with LEC-specific deletion of Foxc1 and Foxc2, alone or in combination, exhibited ERK hyperactivation. Pharmacological ERK inhibition in utero abolished the abnormally enlarged lymphatic vessels in FOXC-deficient embryos. Together, these results identify FOXC1 and FOXC2 as essential regulators of lymphangiogenesis and indicate a new potential mechanistic basis for lymphatic-associated diseases.
\end{abstract}

\section{Introduction}

The lymphatic vasculature system is part of the circulatory system and is responsible for lipid absorption/transport from the digestive tract, maintenance of tissue fluid homeostasis, and immune surveillance (1). This system also has a key role in pathological processes such as inflammatory disease and the metastatic spread of tumor cells $(2,3)$. Lymphatic anomalies include primary (hereditary) and secondary (acquired) lymphedema, and mutations associated with inherited lymphatic malformations have been identified in genes that encode signaling proteins downstream of VEGF-C signaling through its receptor (VEGFR3), including the RAS/ERK pathway $(4,5)$. However, the cause of lymphatic vessel abnormalities in humans is complex and poorly understood.

The development of the lymphatic vascular system in the mouse embryo begins at approximately E9.5, shortly after the establishment of the blood circulation (6). A dorsolateral subpopulation of cells in the anterior cardinal vein becomes competent for differentiation into lymphatic endothelial cells (LECs) when SOX18 and COUP-TFII activate the transcription of Prox1, a master regulator of the lymphatic differentiation and maintenance (7-9). PROX1+ lymphatic endothelial progenitors subsequently bud off, migrate dorsolaterally, and form the primary lymph sacs and superficial lymphatic vessels by E12.5. This process is dependent on VEGF-C expression in the surround-

Conflict of interest: The authors have declared that no conflict of interest exists. Submitted: December 15, 2014; Accepted: April 5, 2016.

Reference information: J Clin Invest. 2016;126(7):2437-2451. doi:10.1172/JCI80465. ing mesenchyme (10). These lumenized lymphatic structures develop into the lymphatic vascular network through the proliferation and sprouting of LECs (developmental lymphangiogenesis). Beginning at E15.5-E16.0, the primary lymphatic vasculature undergoes remodeling and maturation to form a hierarchical lymphatic vascular network composed of lymphatic capillaries and collecting lymphatic vessels (11).

FOXC1 and FOXC2 are closely related members of the FOX (Forkhead box) transcription factor family and have numerous essential roles in cardiovascular development, health, and diseases, including vascular endothelial cell differentiation (12-14). Inactivating mutations in human FOXC2 are responsible for the autosomal dominant syndrome lymphedema-distichiasis, which is characterized by obstruction of lymphatic drainage in the limbs, venous valve failure, and the growth of an extra set of eyelashes $(15,16)$. Lymphatic vessels in individuals with mutations of FOXC2 are hyperplastic (17-19). Recent studies have shown that FOXC2 regulates connexin $37(\mathrm{CX} 37)$ and calcineurin/NFAT signaling during lymphatic valve formation (20-22), as well as lymphatic endothelial cell quiescence (23). Both FOXC1 and FOXC2 play a role in the development of the mouse and human eye (24-28), and mutations or changes in the copy number of human FOXC1 are associated with autosomal-dominant Axenfeld-Rieger syndrome (ARS), which is characterized by anterior eye segment defects, glaucoma, and cerebral small vessel disease $(29,30)$. However, the role of FOXC1 in the lymphatic system has yet to be explored, and it remains to be elucidated how FOXC1 and FOXC2 function in early lymphatic vessel formation. 
In this study, we provide evidence that FOXC1 and FOXC2 are essential factors of lymphatic vessel morphogenesis. In mice, lack of Foxc1, Foxc2, or both genes in LECs leads to increased ERK activation and aberrant LEC proliferation, and abnormally enlarged lymphatic vessels caused by the Foxc1/c2 deletions are rescued by pharmacological inhibition of ERK activation. Collectively, our findings demonstrate a molecular mechanism by which FOXC1 and FOXC2 control lymphatic vessel growth by regulating the Ras/ERK signaling cascade. The identification of a FOXC-mediated molecular axis in this study provides insight into lymphangiogenesis under pathological conditions (3).

\section{Results}

FOXC1 expression in the lymphatic system during mouse development and in human dermal LECs. Previous reports have shown that murine and human FOXC2 are expressed in LECs $(21,31)$; however, evidence of FOXC1 expression in the lymphatic system has been insufficiently investigated, and its role in lymphatic development has yet to be revealed. To this end, we first examined lacZ expression in E10.5 and E12.5 embryos heterozygous for the Foxc1 ${ }^{\text {lac }}$ knock-in allele (24). Consistent with our previous finding that the cardinal vein expresses FOXC1 at E9.5 (32), Foxc1 ${ }^{\text {lacz }}$ was detected in PROX1 ${ }^{+}$LEC progenitors located both in the cardinal vein at E10.5 and in cells budding from it (Figure 1A), and in PROX1 $1^{+}$LECs located in the lymph sacs at E12.5 (Figure 1B). Next, we sought to determine spatiotemporal expression patterns of murine FOXC1, compared with FOXC2, in the developing lymphatic system (Figure 1, C-F). In line with the Foxc1 $1^{\text {lacz }}$ expression patterns described above, triple immunostaining of FOXC1, FOXC2, and PROX1 revealed that FOXC1 and FOXC2 were coexpressed in PROX1 ${ }^{+}$LEC progenitors at E10.5 and E12.5 (Figure 1, C and D). Furthermore, we confirmed that transcriptional levels of Foxc1 were comparable to those of Foxc2 and Prox1 in PROX1 ${ }^{+}$LYVE-1 ${ }^{+}$LECs isolated from E15.5 dorsal skin (Figure $1 G)$. Expression of FOXC2 and PROX1 is upregulated in early lymphatic valve-forming cells of collecting lymphatic vessels (beginning at $\sim \mathrm{E} 15.5$ ), an initial process to define the valve territory (20, 22). Immunostaining of mesenteric lymphatic vessels at E17 and $\mathrm{P} 3$ revealed that FOXC1 was colocalized with FOXC2 and PROX1 in the valve-forming cells (Figure 1, E and F).

In line with the expression patterns in the developing lymphatic vasculature, human FOXC1 and FOXC2 transcripts were both detected in neonatal dermal microvascular LECs (Figure $1 \mathrm{H})$, which is in agreement with a previous microarray analysis of freshly isolated cutaneous human LECs (33). Collectively, these data indicate that FOXC1 and FOXC2 show overlapping expression patterns during lymphatic vessel development, and that like FOXC2, FOXC1 is likely to function in the lymphatic system.

Global deletion of Foxc1 in mice results in abnormal lymphatic vessel morphogenesis. To determine the role of murine FOXC1 in the lymphatic vasculature system, we first analyzed global Foxc1 $1^{\text {lacz/lacz }}$ mutant (global-Foxc1-KO) embryos (24). At E15.5, they displayed edema in the dorsal skin (Figure 2B, arrowheads), as previously reported (24). Abnormal lymphatic vessel morphogenesis was observed in the dorsal skin of the global-Foxc1-KO embryos (Figure $2 \mathrm{H}$, arrow), whereas formation of large blood vessels in these mutants appeared normal in the dorsal skin (Figure $2 \mathrm{~F}$ ), as well as the facial and hind limb regions (Figure 2, B and D, arrows). We further carried out morphometric analysis of lymphatic vessel width (LVW) and branch points in LYVE-1-immunostained E15.5 dermal lymphatic vessels (Figure 2, I and J). Lymphatic vessels were significantly enlarged in the global-Foxc1-KO embryos (Figure 2I), but there was no apparent difference in the number of lymphatic branch points between the global-Foxc1-KO and WT embryos (Figure 2J). These results suggest that global deletion of Foxc1 leads to lymphatic vessel abnormalities in the developing skin.

LEC-specific deletion of Foxc1 and Foxc2 in mice results in increased LEC proliferation, leading to enlarged lymphatic vessels. Similar to our analysis of the global-Foxc1-KO mice, previous studies on the role of Foxc2 in the lymphatic vessel system have employed global Foxc2-knockout mice (20-22). To determine the specific functions of FOXC1 and FOXC2 in the cellular processes during early lymphatic development, we crossed conditional-null Foxc1 ${ }^{f l}$ and Foxc $^{f l}$ mutant mice (34) with Prox1-CreER ${ }^{T 2}$ mice (35) to generate tamoxifen-inducible, LEC-specific Foxc1-mutant (Prox1-CreER ${ }^{T 2}$ Foxc1 $^{f / f l}$; referred to herein as LEC-Foxc1-KO), Foxc2-mutant (Prox1-CreER ${ }^{T 2}$ Foxc $2^{f / f l}$; LEC-Foxc2-KO), and compound Foxc1;Foxc2-mutant (Prox1-CreER ${ }^{T 2}$ Foxc1 $^{f l / f l}$ Foxc2 ${ }^{f / f l}$; LEC-Foxc1;Foxc2-DKO) mice. Pregnant dams were injected with tamoxifen at E10.5 to induce the mutations, and we confirmed that this method of CreER ${ }^{\mathrm{T} 2}$ induction efficiently deleted both Foxc1 and Foxc2 genes in the lymph sacs at E12.5 (Supplemental Figure 1, A-F; supplemental material available online with this article; doi:10.1172/JCI80465DS1) and the dorsal skin at E15.5 (Supplemental Figure 1, G-I). We initially investigated the formation at E12.5 of the primary lymph sacs, as they are the first lumenized lymphatic structures created by coalescence of PROX1 $1^{+}$LEC progenitors derived from the cardinal vein and intersomitic vessels (36). By PROX1 immunostaining to detect the jugular lymph sacs, appreciably more $\mathrm{PROX}^{+} \mathrm{LECs}$ were found in all three lines of LEC-Foxc-KO embryos than in their control littermates (Figure 3, A-F). Quantitative analysis verified a significant increase in the number of PROX $1^{+}$LECs in the lymph sacs of the single and double LEC-Foxc mutants (Figure 3, G-I). Consequently, the Foxc deletions resulted in abnormally enlarged jugular lymph sacs.

At E12.5, the proliferation rate of LECs in the lymph sacs was significantly enhanced in all three lines of LEC-Foxc-KO embryos (Figure 3, J-R). There was little or no apoptotic activity in the lymph sacs detected by TUNEL assay in the Foxc mutant and littermate control embryos (Supplemental Figure 3). Collectively, these observations indicate that LEC-specific loss of Foxc1, Foxc2, or both in mice increases LEC proliferation. Our findings are also in agreement with reports that individuals with inactivating mutations in FOXC2 have hyperplastic lymphatic vessels (17-19).

LEC-specific ablation of Foxc1 and Foxc2 leads to abnormal lymphatic vessel morphogenesis and dermal edema. We analyzed lymphatic vessel morphogenesis at E15.5 in the dorsal skin of all three lines of LEC-Foxc-KO embryos (Figure 4). Although no apparent edema was observed in either LEC-Foxc1-KO or LEC-Foxc2-KO embryos (Figure 4, A and F), close examination of histological sections revealed subcutaneous edema in the LEC-Foxc1-KO and LEC-Foxc2-KO embryos (Figure 4, B and G). Importantly, the LEC-Foxc1-KO and LEC-Foxc2-KO embryos displayed abnormally enlarged lymphatic vessels in the dorsal skin (Figure 4, $\mathrm{C}$ and $\mathrm{H}$, 

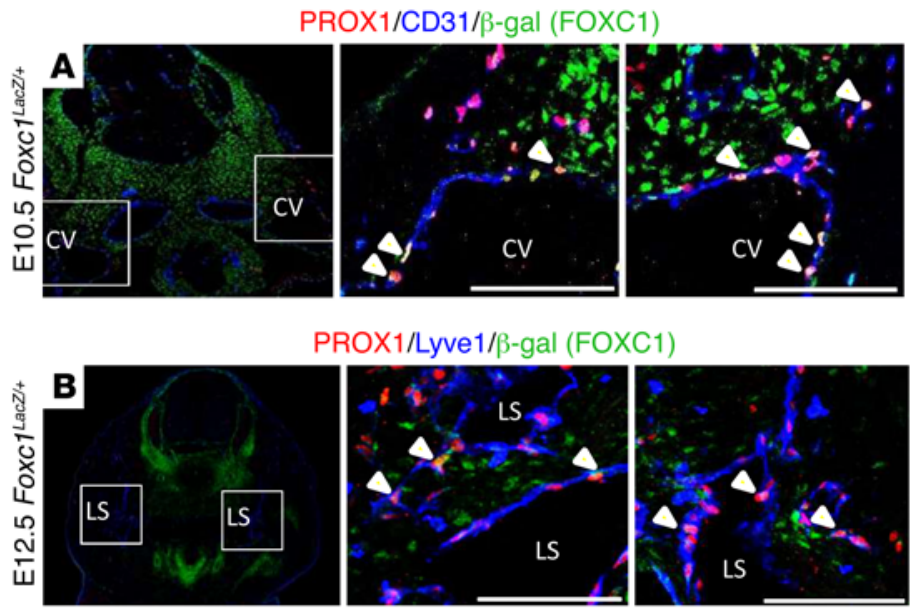

PROX1/Lyve1//-gal (FOXC1)

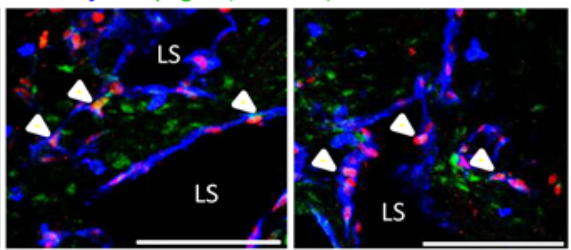

G

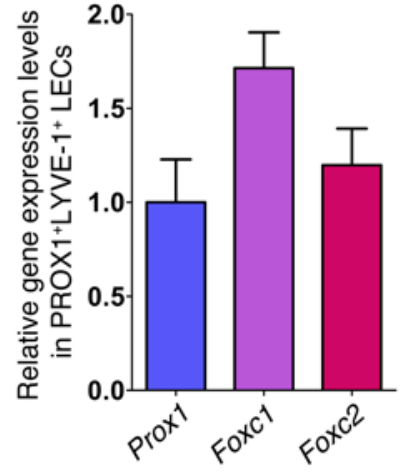

H
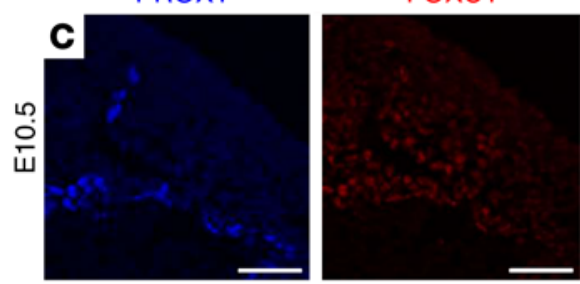

FOXC2
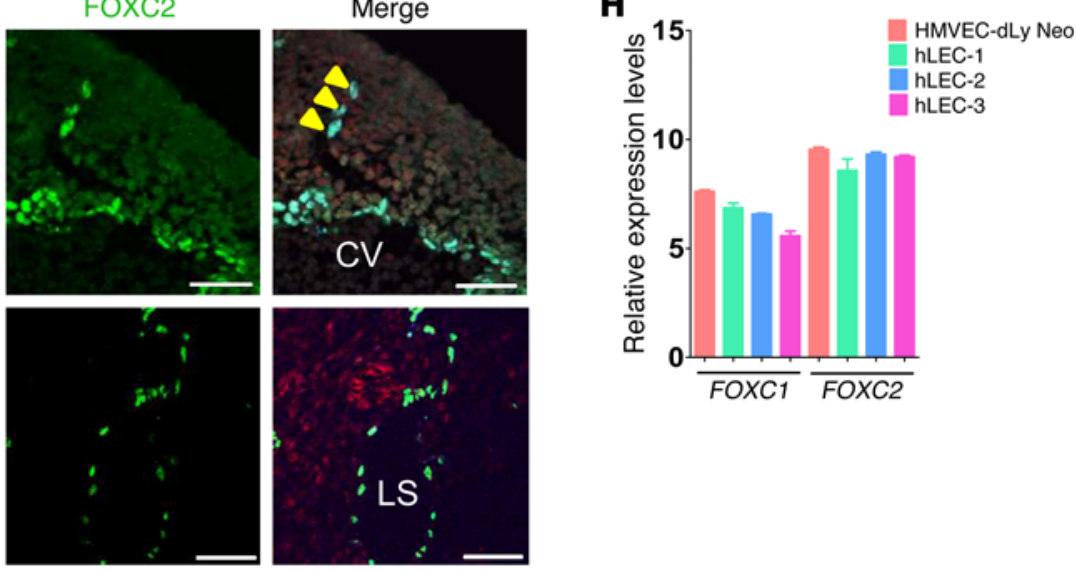
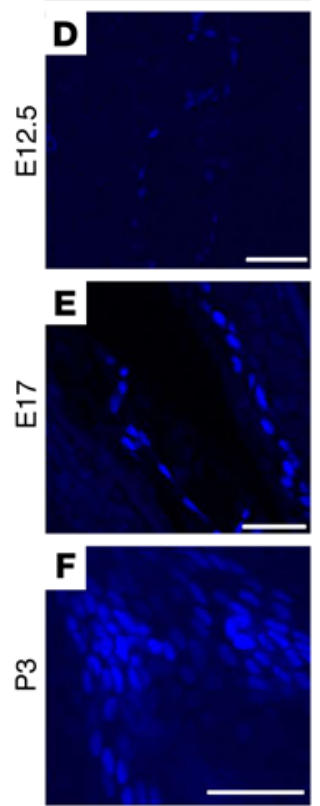
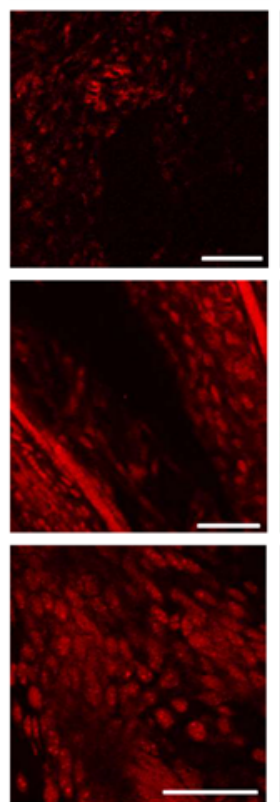
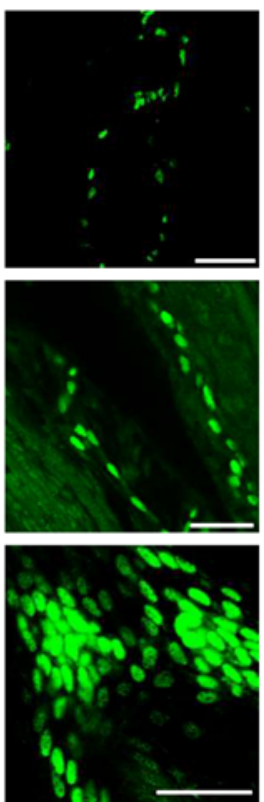
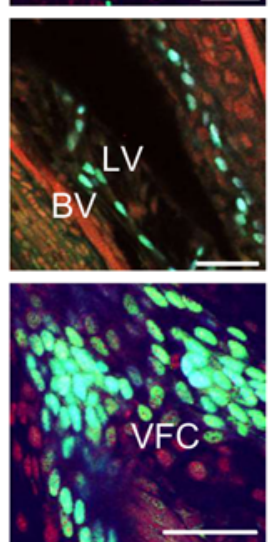

Figure 1. FOXC1 and FOXC2 are coexpressed in LEC progenitors and lymphatic valve-forming cells during mouse development. (A and B) Foxc1 expression in PROX1+ LEC progenitors of FoxC 1acz/+ embryos as revealed by $\beta$-gal immunostaining. The middle and right panels show a higher magnification of the boxed regions indicated in the left panels $(\times 40)$. (A) Triple immunostaining of PROX1, CD31, and $\beta$-gal (FOXC1) at E10.5. PROX1+ $\beta$-gal ${ }^{+}$LEC progenitors were detected in the cardinal vein (CV) and cells migrating out of the CV (white arrowheads). Scale bars: $100 \mu$ m. (B) Triple immunostaining of PROX1, LYVE-1, and $\beta$-gal (FOXC1) at E12.5. PROX1+ $\beta$-gal ${ }^{+}$LECs were detected in lymph sacs (LS) (white arrowheads). Scale bars: $100 \mu \mathrm{m}$. (C) Triple immunostaining of FOXC1, FOXC2, and PROX1 on an E10.5 sagittal section. FOXC1 and FOXC2 were coexpressed in PROX1-expressing LEC progenitors in the CV and cells migrating out of the CV (yellow arrowheads). (D) Triple immunostaining of FOXC1, FOXC2, and PROX1 at the level of the neck on an E12.5 longitudinal section. FOXC1 and FOXC2 were detected in PROX1-positive LS. (E and F) Whole mount triple immunostaining of mesenteric vessels at E17 (E) and P3 (F) for FOXC1, FOXC2, and PROX1. Note overlapping expression of FOXC1, FOXC2, and PROX1 in lymphatic valve-forming cells (VFCs). BV, blood vessel; LV, lymphatic vessel. Scale bars: $50 \mu \mathrm{m}$. (C) qPCR analysis of relative mRNA levels of Foxc1, Foxc2, and Prox1 in PROX1+LYVE-1+ LECs isolated from E15.5 dorsal skin. The value of Prox1 mRNA levels is set at 1-fold. (H) qPCR analysis of relative mRNA levels of FOXC1 and FOXC2 in human LECs isolated from foreskin samples of donors between 2 and 5 years of age (HLEC-1, HLEC-2, and HLEC-3) and human neonatal LECs from Lonza (HMVEC-dLy Neo). 


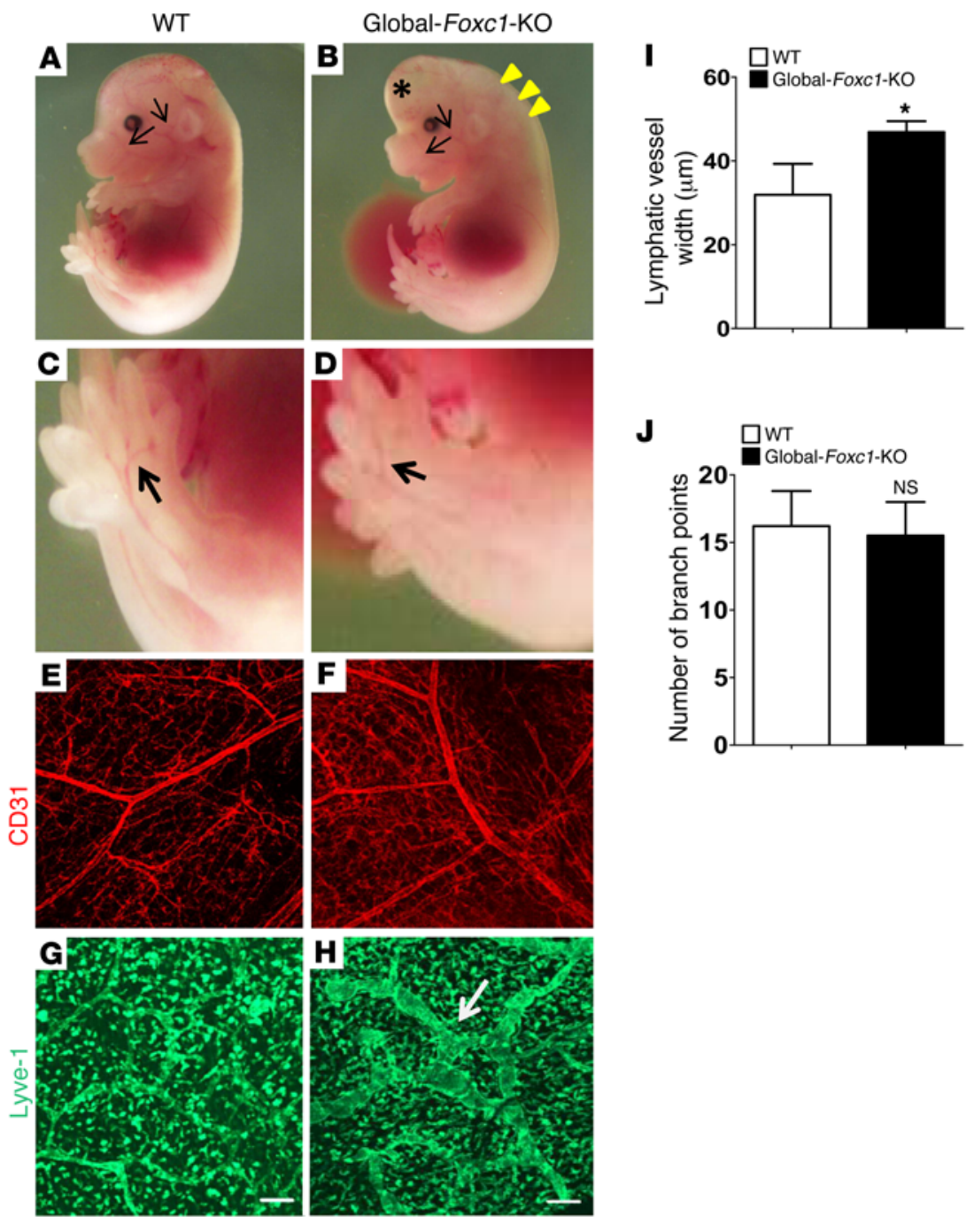

Figure 2. Global deletion of Foxc1 results in lymphatic vessel abnormalities. (A-D) Hydrocephalus (asterisk) and subcutaneous edema (arrowheads) in globalFoxc1-KO embryo (B) at E15.5. Arrows indicate blood vessel formation in the facial and hind limb regions. ( $E$ and $\mathbf{F}$ ) Whole mount CD31 immunostaining of the dorsal skin at E15.5. (G and $\mathbf{H}$ ) Whole mount LYVE-1 immunostaining of the dorsal skin at E15.5. ClobalFoxc1-KO embryos exhibited dilated lymphatic vessels (arrow) compared with WT littermates. (I and J) Morphometric analysis of LVW (I) and the number of lymphatic branch points (J) in LYVE-1-immunostained E15.5 dorsal skins. $P$ values were obtained by 2 -tailed Student's $t$ test. Data are presented as mean \pm SEM. $n=3 .{ }^{*} P<0.05$. Scale bars: $100 \mu \mathrm{m}$.

embryos at E15.5 was significantly downregulated (Figure 4Q and Supplemental Table 3). Immunohistological analysis of the distribution of VE-cadherin in LECs confirmed that lymphatic endothelial cell-cell junctions (38) were disrupted in the dorsal skin of the LEC-Foxc-KO embryos (Figure 4, R-T). These results suggest that lack of the Foxc1/c2 genes reduces vascular barrier function and junction integrity in LECs.

We also analyzed LEC proliferation and survival in the dorsal skin of single and double LEC-specific Foxc mutants at E15.5. None of the three lines of LEC-Foxc mutant embryos showed significant changes in LEC proliferation compared with their littermate controls at this embryonic stage (Supplemental Figure 4, A-C), probably because lymphangiogenesis in the dorsal skin appeared to have reached a plateau. On the other hand, the Foxc-mutant lymphatic vessels exhibited decreased apoptotic activity

arrows). Morphometric analysis also indicated that the Foxc-mutant lymphatic vessels were much wider than normal (Figure 4, D and I), but they did not show any difference in branching pattern (Figure 4, E and J).

We next analyzed the lymphatic vessel vasculature in LECFoxc1;Foxc2-DKO embryos. Specifically, severe edema was present in approximately $5 \%$ of the LEC-Foxc1;Foxc2-DKO embryos at E15.5 (Figure 4L, arrowheads), which was not observed in any of the single LEC-Foxc-KO embryos examined. Like in the single LEC-Foxc-KO embryos described above, the lymphatic vessels in the dorsal skin were abnormally shaped and widened in the LECFoxc1;Foxc2-DKO embryos (Figure 4, N and O); however, these mutant vessels showed a normal branching pattern (Figure 4P). To identify potential molecular components of the mechanisms by which FOXC1 and FOXC2 regulate lymphatic vessel development, we performed our recently developed genome-wide RNAseq transcriptome analysis (37) with RNA extracted from sorted LECs that had been isolated from the dorsal skin of compound LEC-Foxc1;Foxc2-DKO embryos and their littermate controls at E15.5 (Supplemental Figure 2 and Supplemental Tables 1-3). RNAseq analysis showed that expression of VE-cadherin (Cdh5) in LECs isolated from the dorsal skin of the LEC-Foxc1;Foxc2-DKO
(Figure 4, U-X, and Supplemental Figure 4, D-H). Together, these data indicate that the specific loss of Foxc1, Foxc2, or both in LECs leads to abnormal formation of lymphatic vessels.

Foxc1 and Foxc2 regulate the Ras/ERK pathway during lymphatic vessel morphogenesis. Our results of RNA-seq analysis indicated that suppressors of Ras function (Ras GTPase-activating protein [RasGAP]; Rasa4 and Rasal3) were significantly downregulated in the Foxc1/c2-double mutant LECs (Figure 5A). Notably, mutations in KRAS, HRAS, and the RasGTPase p120RASGAP (RASA1) that lead to increased Ras activity are associated with primary lymphedema and lymphatic malformations in humans (4), which is further supported by findings from several mouse models $(39,40)$. By aligning human and mouse genomic sequences containing the RASA4 and RASAL3 loci (promoter and exon/intron regions) with the ECR (Evolutionary Conserved Regions) Browser (http://ecrbrowser.dcode.org), we identified highly conserved FOXC-binding sites $(20,41)$ in these loci (Figure $5, \mathrm{~B}$ and $\mathrm{C}$ ). To further define the molecular nature of FOXC function in these binding sites, human dermal LECs (HMVECdlyNeo) were cultured, and ChIP assays was performed using two specific antibodies for each FOXC protein (Figure 5, D and E, and Supplemental Figure 5A). We found that both FOXC1 

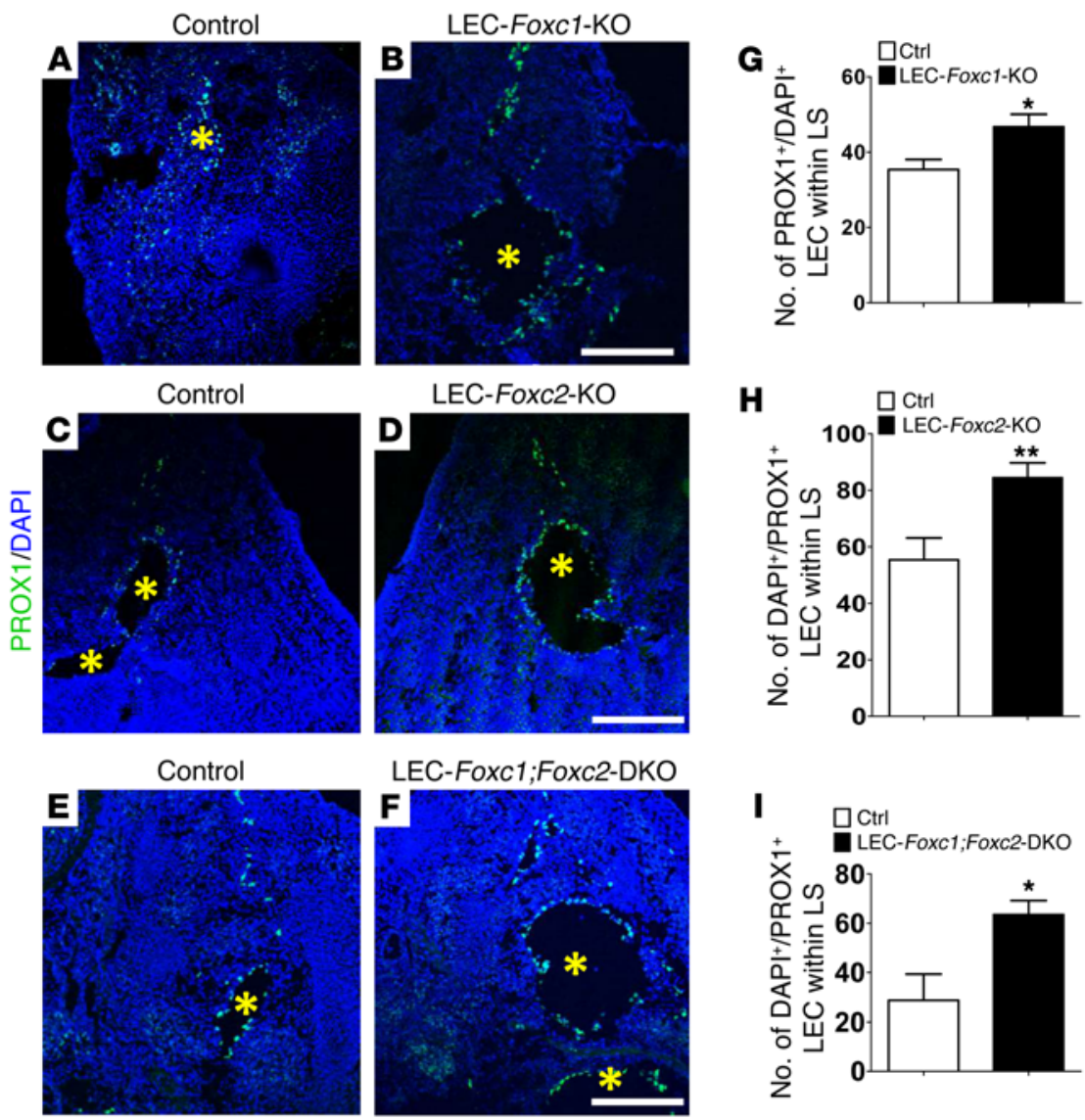

Figure 3. LEC-specific deletion of Foxc1 and Foxc2 leads to increased LEC specification and LEC hyperplasia. (A-F) PROX1 immunostaining of E12.5 lymph sacs (asterisks). LEC-Foxc1-KO (B), LEC-Foxc2-KO (D), and LEC-Foxc1;Foxc2DKO (F) embryos displayed increased numbers of PROX1+ LECs compared with littermate controls (A, C, and E). Scale bars: $100 \mu \mathrm{m}$. (G-I) Quantification of PROX1+ LECs in E12.5 lymph sacs. Data are presented as mean \pm SEM. $n=4$. ${ }^{*} P<0.05,{ }^{*} P<0.01$. (J-0) BrdU staining of LYVE-1+ LECs in E12.5 lymph sacs. Arrowheads indicate BrdU+ cells. Low-magnification insets $(\times 4)$ highlight differences in the size of lymph sacs between control and mutant embryos. Scale bar: $50 \mu \mathrm{m}$. (P-R) Quantification of BrdU+ LECs in E12.5 lymph sacs. $P$ values were obtained by 2 -tailed Student's $t$ test. Data are presented as mean \pm SEM. $n=7$. ${ }^{*} P<0.05,{ }^{* *} P<0.01$.

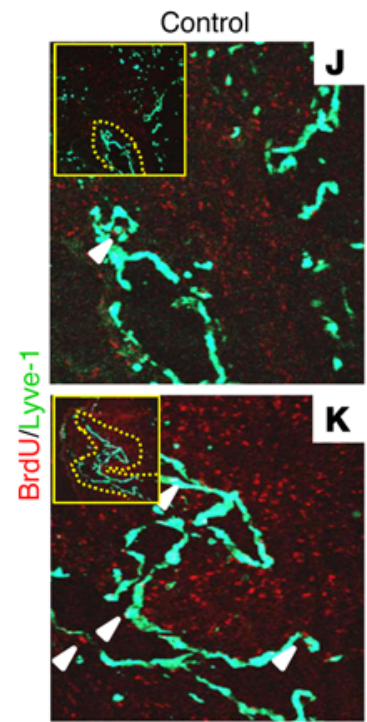

LEC-Foxc1-KO
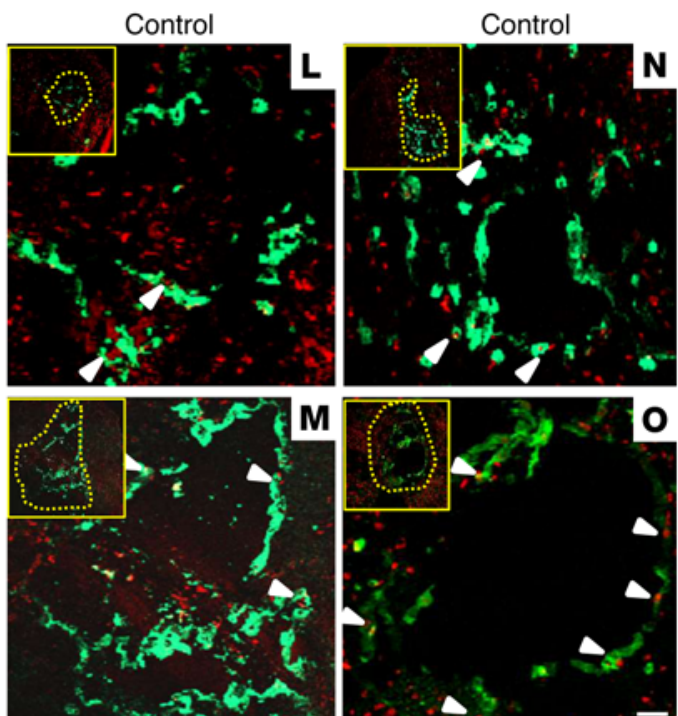

LEC-Foxc2-KO

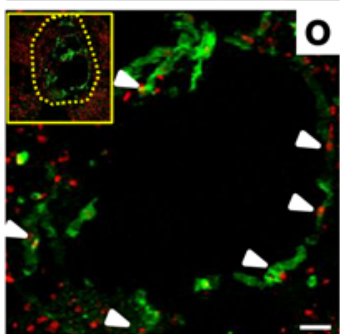

LEC-Foxc1;Foxc2-DKO
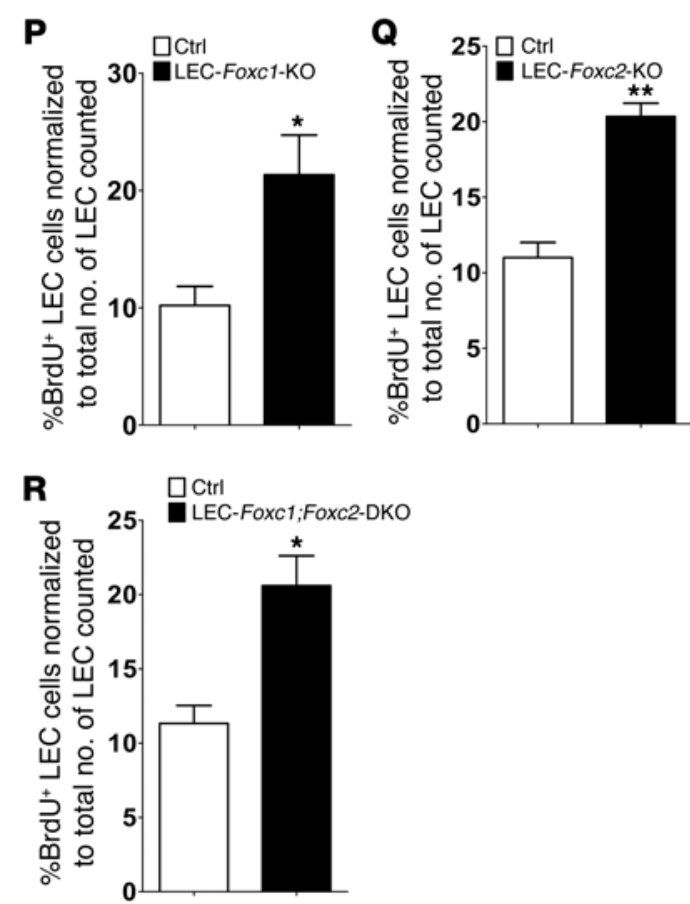

and FOXC2 commonly bound to ECR6, -10, and -17 in RASA4. FOXC1 and FOXC2 bound to ECR2/5 and ECR17, respectively, in RASAL3, while specific binding of FOXC1 and FOXC2 to RASAL3-ECR13/17 and RASAL3-ECR2/5/13, respectively, was detected by one of the two ChIP antibodies used. The variance of
ChIP signals between the two antibodies against each FOXC protein may be due, at least in part, to different epitopes recognized by them. These results indicate that FOXC1 and FOXC2 have similar binding capacities for the RASA4 and RASAL3 loci. We also found that FOXC2 similarly bound to RASA4-ECR6/10/17 

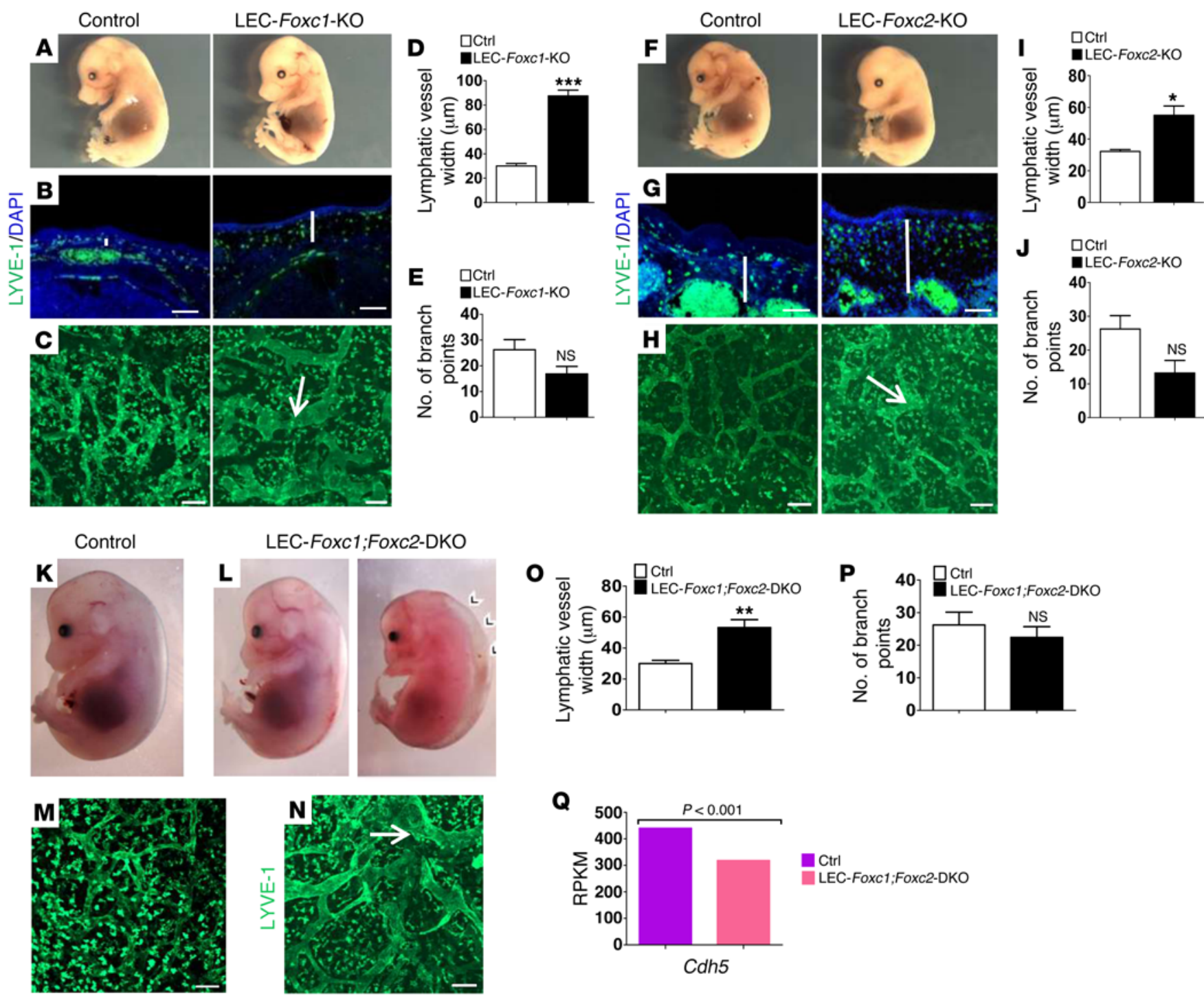

ECtrl
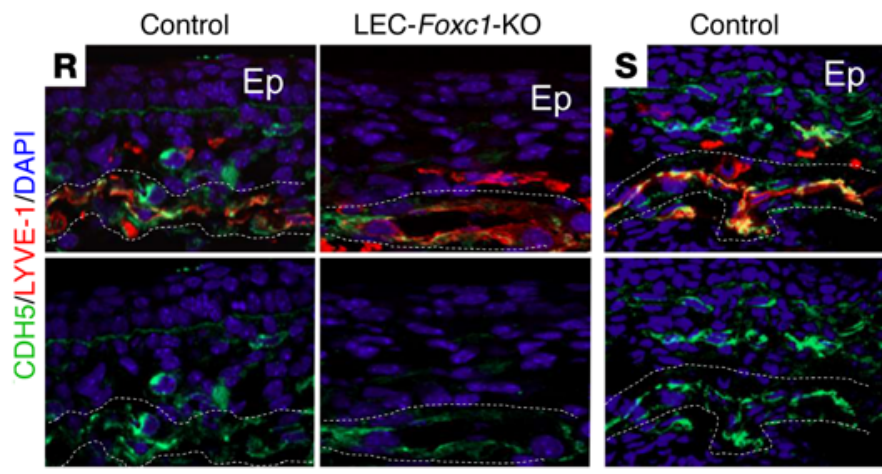

LEC-Foxc2-KO
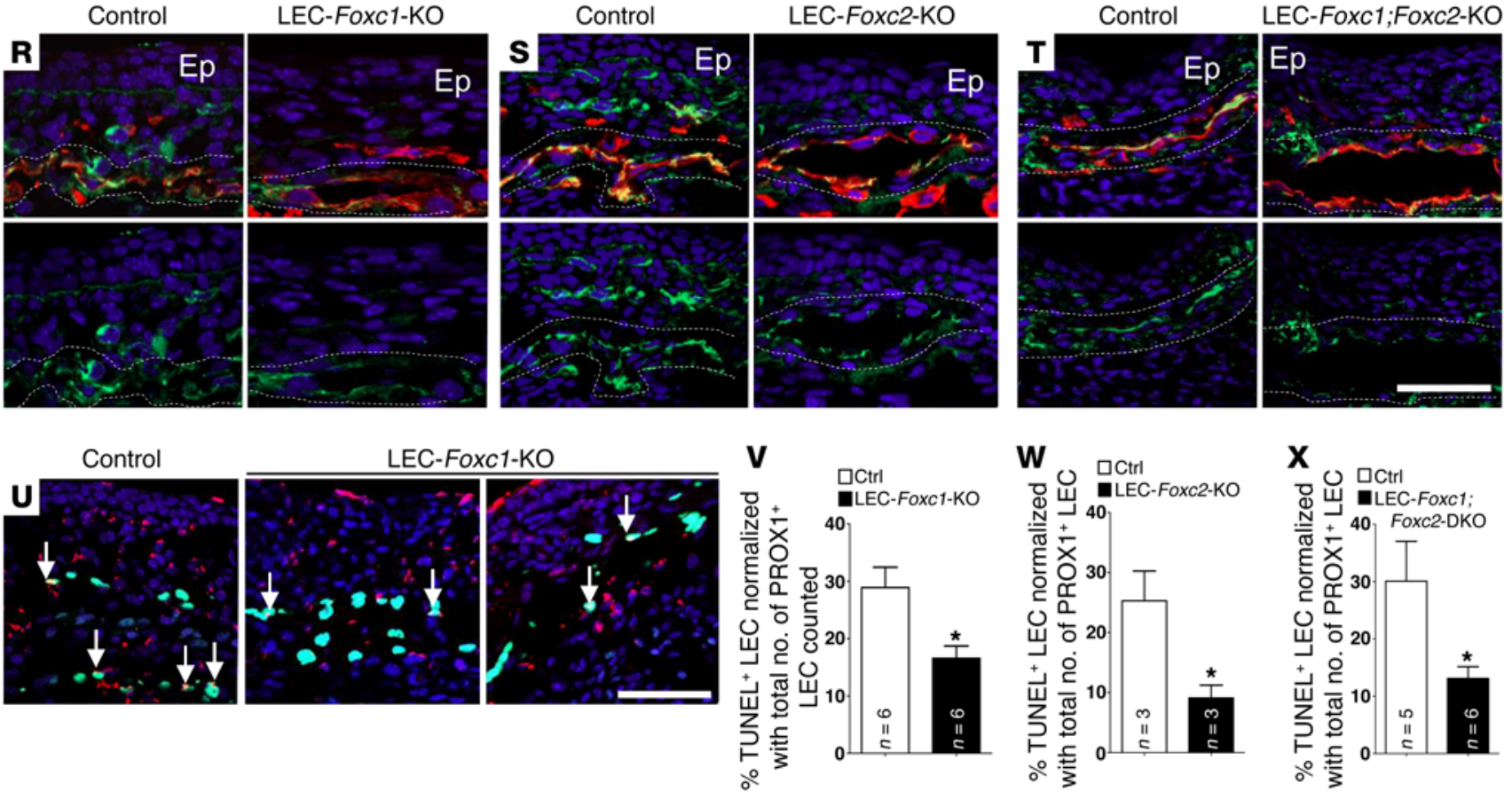

$\mathbf{X}$

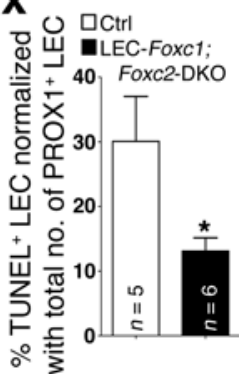


Figure 4. LEC-specific ablation of Foxc1 and Foxc2 results in abnormal lymphatic vessel formation. (A) Gross morphological analysis of E15.5 LEC-FoxC1-KO embryos. (B) LYVE-1 immunostaining of E15.5 dorsal skins reveals enlarged dermis (marked by white line) in LEC-Foxc1-KO embryo. (C) Whole mount LYVE-1 immunostaining of E15.5 dorsal skins showing larger lymphatic vessels (arrow) in LEC-Foxc1-KO embryo. (D and E) Morphometric analysis of LVW (D) and the number of lymphatic branch points (E) in LYVE-1-immunostained dorsal skins at E15.5. Data are presented as mean \pm SEM. $n=5$. ${ }^{* *} P<0.001$. (F) Gross morphological analysis of E15.5 LEC-Foxc2-KO embryos. (G) LYVE-1 immunostaining of E15.5 dorsal skins reveals enlarged dermis (marked by white line) in LEC-Foxc2-KO embryo. (H) Whole mount LYVE-1 immunostaining of E15.5 dorsal skins showing larger lymphatic vessels (arrow) in LEC-Foxc2-KO embryo. (I and J) Morphometric analysis of LVW (I) and the number of lymphatic branch points (J) in LYVE-1-immunostained E15.5 dorsal skins. Data are presented as mean \pm SEM. $n=5$. ${ }^{*} P<0.05$. ( $\mathbf{K}$ and $\mathbf{L}$ ) Gross morphological analysis of LECFoxc1;Foxc2-DKO embryos. A small number of LEC-Foxc1;Foxc2-DKO embryos (5\%) exhibited severe subcutaneous edema (arrowheads). (M and N) Whole mount LYVE-1 immunostaining of E15.5 dorsal skins showing larger lymphatic vessels (arrow) in LEC-Foxc1; Foxc2-DKO embryo. (0 and P) Morphometric analysis of LVW (0) and the number of lymphatic branch points $(\mathbf{P})$ in LYVE-1-immunostained E15.5 dorsal skins. $P$ values were obtained by 2-tailed Student's $t$ test. Data are presented as mean \pm SEM. $n=5$. ${ }^{* *} P<0.01$. (Q) Decreased Cdh5 expression in LECs isolated from the dorsal skin of LEC-Foxc1; Foxc2-DKO embryos at E15.5. Graph shows RPKM (reads per kilobase of coding sequence per million mapped) values from RNA-seq. (R-T) Immunostaining of E15.5 dorsal skins for CDH5 and LYVE-1 showing disruption of lymphatic endothelial cell-cell junctions in LECFoxc-KO embryos. Five controls and 5 mutants were examined for each mutant line. Ep, epithelium. Scale bar: $100 \mu \mathrm{m}$. (U-X) Immunostaining of E15.5 dorsal skins for PROX1 combined with TUNEL assay. (U) Representative images of control and LEC-FoxC1-KO embryos. Arrows indicate PROX1+TUNEL ${ }^{+}$LECs. (V-X) Quantification TUNEL ${ }^{+}$LECs in LEC-FoxC1-KO (V), LEC-Foxc2-KO (W), and LEC-Foxc1;Foxc2-DKO (X) embryos compared with littermate controls. $P$ values were obtained by 2 -tailed Student's $t$ test. Data are presented as mean \pm SEM. ${ }^{*} P<0.05$. Scale bars: $50 \mu \mathrm{m}$

and RASAL3-ECR5/17 in HUVECs (Supplemental Figure 5, $\mathrm{B}$ and $\mathrm{C})$. Knockdown of FOXC2 significantly increased Ras activity in HUVECs at baseline (without VEGF), whereas VEGF stimulation in control cells appeared to activate Ras to a similar extent as in FOX2-deficient cells (Figure 5, F and G).

To test whether LEC-Foxc-KO embryos display hyperactivation of the ERK pathway, we performed double immunostaining for PROX1 and phosphorylated ERK1/2 (p-ERK1/2), because p-ERK is a measurable end point of activated Ras signaling. The number of p-ERK ${ }^{+}$LECs in the lymph sacs at E12.5 was significantly increased in all three lines of LEC-Foxc-KO embryos by 2- to 3-fold (Figure 6). Similarly, global Foxc2 $2^{+-}$embryos exhibited enhanced ERK activity in the lymph sacs (Figure 6, F and K). To further investigate the relationship between the ERK hyperactivation and lymphatic defects in LEC-Foxc-KO embryos, we tested whether and to what extent the abnormal lymphatic phenotypes associated with the Foxc1/c2 deletions could be rescued in the embryos by injecting the MEK inhibitor U0126 (42) into female mice that were pregnant with either single or double LEC-Foxc-KO embryos at E12.5 and E13.5. As shown in Figure 7, A-F, U0126 treatment inhibited aberrant activation of ERK in Foxc-mutant LECs of the dorsal skin at E14.5, and the abnormal proliferative capacity of LECs in Foxc mutants was also abolished (Figure 7, G-I). Most importantly, U0126 treatment reduced LVW in all three lines of
LEC-Foxc-KO embryos at E15.5, completely abolishing lymphatic vessel dilation compared with their littermate controls (Figure 7, $\mathrm{J}-\mathrm{X})$. Treatment with another MEK inhibitor, PD0325901 (43), also effectively rescued the abnormally enlarged lymphatic vessels in all three lines of LEC-Foxc-KO embryos (Supplemental Figure 6). Taken together, these results suggest that deficiencies of Foxc1 and Foxc2 result in increased ERK activation, leading to the overgrowth of lymphatic vessels.

FOXC1 plays a role in lymphatic vessel development. Recent studies have begun to characterize the complex process of lymphatic valve formation and maturation $(44,45)$. FOXC2 and PROX1 are initially upregulated in clusters of lymphatic valve-forming cells $(20,45)$ and act in concert with mechanical forces to establish the territory for valve formation by regulating $C \times 37$ expression (22). Despite the presence of FOXC1 expression in lymphatic valveforming cells at E17 and P3 (Figure 1, E and F), the role of FOXC1 in the lymphatic valves has yet to be determined. After tamoxifen-induced Cre-mediated recombination at E10.5, valve-forming PROX ${ }^{\text {hi }}$ LECs clustered and reoriented in littermate control mice by at least $45^{\circ}$ at E18.5, as described previously $(22,44,46)$ (Supplemental Figure 7, B and E). In contrast, the clustering and reorientation of PROX $1^{\text {hi }}$ LECs in LEC-Foxc1-KO embryos was significantly disrupted (Supplemental Figure 7, D and E).

Mice with an endothelial cell-specific (EC-specific) Foxc1 mutation (EC-Foxc1-KO) $(47,48)$, generated by crossing Foxc $1^{l}$ mice (34) with Tie2-Cre mice (49), were also examined for defects in mesenteric lymphatic valves, as shown in the deletion of the integrin- $\alpha 9$ gene in the developing lymphatic valves (50) using Tie2-Cre mice (51). According to recent descriptions of the stages of lymphatic valve development $(22,44,52)$, the proportion of mature lymphatic valves that had formed a characteristic V-shape at P7 was significantly lower in the EC-Foxc1-KO mice than in their littermate controls (Supplemental Figure 7, F-K). This observation accords with the defective formation of lymphatic valves in the LEC-Foxc1-KO mice described above. Importantly, FOXC2 appeared normally expressed in the valve-forming cells of the EC-Foxc1-KO embryos, indicating that Foxc1 and Foxc2 do not regulate each other in these cells, as previously shown in aortic arch arteries (53). These data demonstrate that Foxc1 deficiency impairs valve maturation.

\section{Discussion}

Despite recent progress toward deciphering the cellular and molecular mechanisms underlying the formation of the lymphatic vascular system, understanding of this highly orchestrated, complex process remains largely limited. In this study, we demonstrate, for the first time to our knowledge, that FOXC1 and FOXC2 are required for regulation of the Ras/ERK signaling pathway in lymphangiogenesis. Given evidence that the genetic cause of lymphatic disorders is still unidentified in the majority of familial and sporadic cases (54), this finding expands our knowledge of lymphatic vessel formation and provides insight into other disease processes that are associated with pathological lymphangiogenesis (3).

We demonstrate that the loss of Foxc1, Foxc2, or both in LECs leads to ERK hyperactivation (Figures 6 and 7), which accords with the increased LEC proliferation (Figure 3) and abnormal patterning of lymphatic vessels (Figure 4). Previous studies show that 


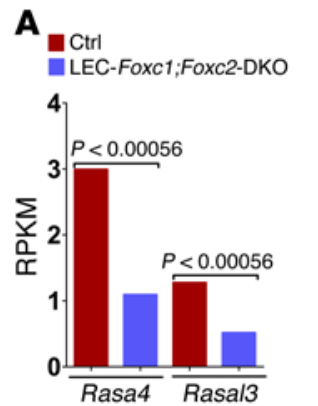

B RASA4

UCSC genome browser on human Feb 2009 (GRCh37/hg19) assembly

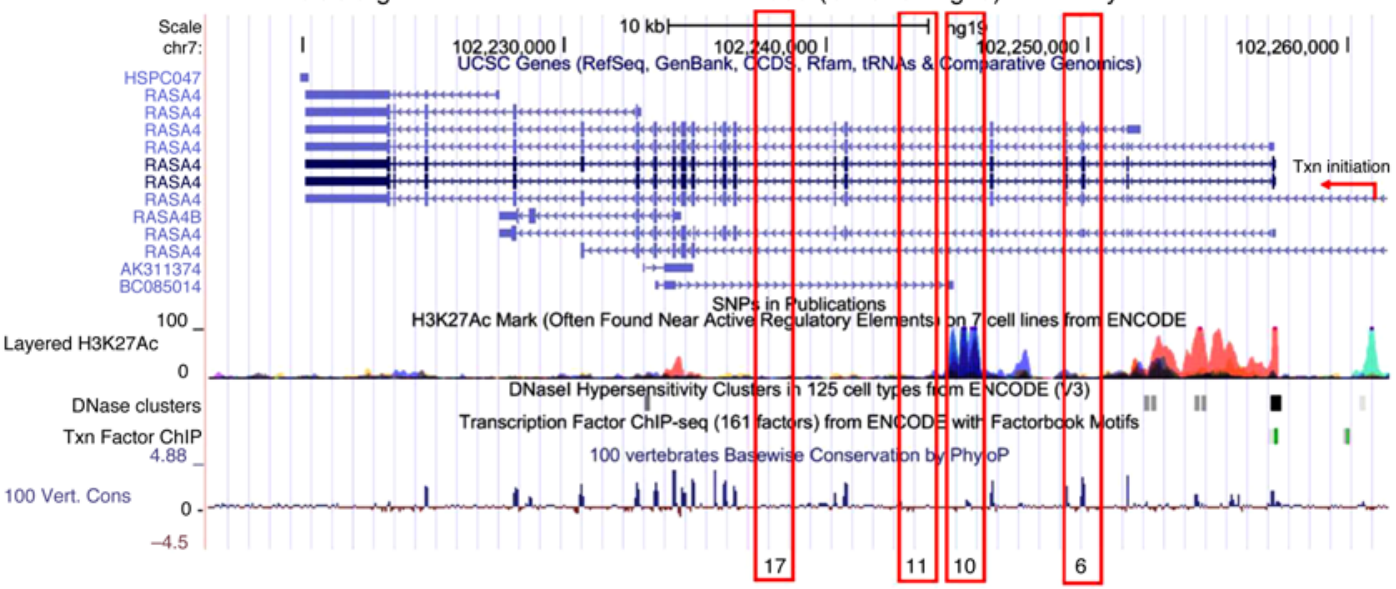

C RASAL3

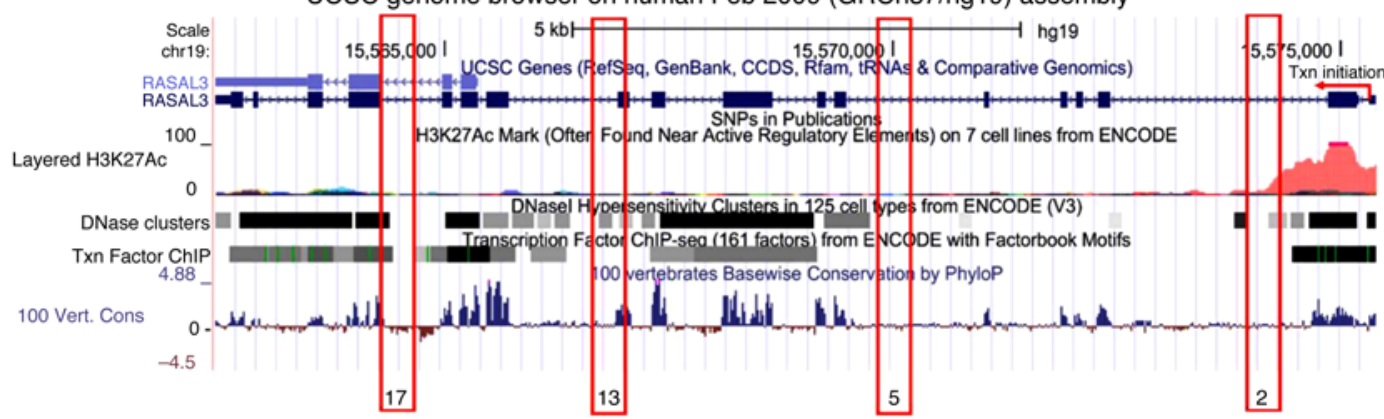

D
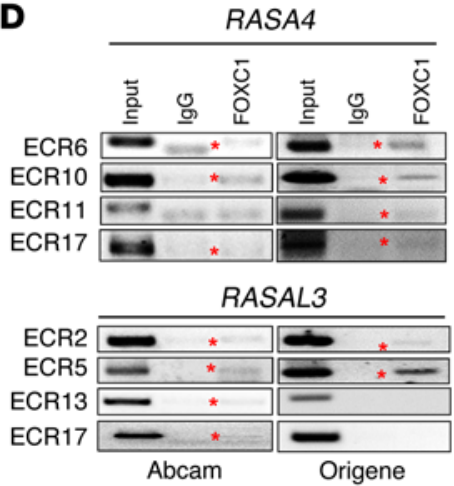

E

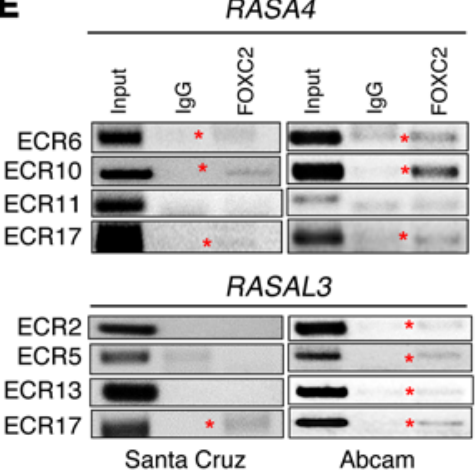

$\mathbf{F}$

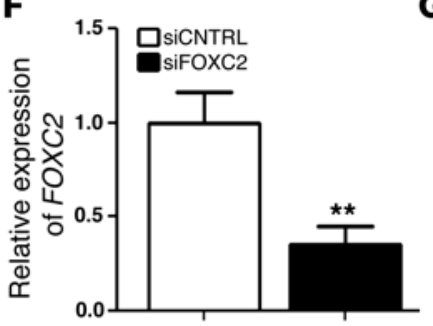

G

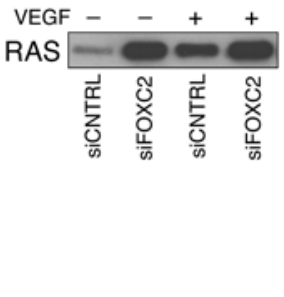

Figure 5. FOXC1 and FOXC2 regulate lymphatic vessel development by controlling the Ras/ERK signaling pathway. (A) Reduced expression of Rasa4 and Rasal3 in Foxc1/c2-double mutant LECs isolated from the dorsal skin at E15.5. Graphs show RPKM values from RNA-seq analysis. (B and C) Putative FOXC-binding sites in the human RASA4 (B) and RASAL3 (C) loci, as viewed on the UCSC Human Cenome Browser (http://genome.ucsc.edu; ref. 71). Red boxes indicate evolutionary conserved regions (ECRs) containing FOXC-binding sites between human and mouse. (D and E) ChIP showing specific binding of FOXC1 (D) and FOXC2 (E) to the consensus FOXC-binding sites within ECRs in RASA4 and RASAL3 in human LECs (HMVEC-dLyNeo). Asterisks indicate FOXC-specific binding. Note that specific antibodies against FOXC1 (left, Abcam; right Origene) and FOXC2 (left, Santa Cruz Biotechnology Inc.; right, Abcam) were used in $\mathbf{C}$ and $\mathbf{D}$, respectively. (F) qPCR analysis of relative mRNA levels of FOXC2 in HUVECs transfected with negative control siRNA or siRNA targeting FOXC2. $P$ values were obtained by 2 -tailed Student's $t$ test. Data are reported as mean \pm SEM. $n=3$. ${ }^{*} P<0.01$. (G) HUVECs transfected with negative control or FOXC2 siRNA were serum deprived and stimulated with VEGF where indicated prior to lysis and pull down of GTP-associated Ras. Detection of active Ras pull down was performed by Western blotting, and a representative image of 3 separate experiments is shown.

Foxc1 and Foxc2 play overlapping roles in mouse development (32, $53,55)$, whereas increased ERK activation and lymphatic vessel growth in Foxc1/c2-deficient LECs did not particularly show additive effects of loss of both genes. One possible explanation is that lack of one of the two genes may have reached a plateau in the maximum ERK activation in the developing lymphatic vasculature.
Our study identifies Ras regulators (RASA4 and RASAL3) as downstream genes of FOXC1 and FOXC2 in LECs (Figure 5). RASA1, RASA4, and RASAL3 are all members of the RasGAP protein family that share a conserved GAP domain; therefore, it is plausible that they have redundant functions in the regulation of Ras activity during lymphatic vessel formation. It should be noted that 

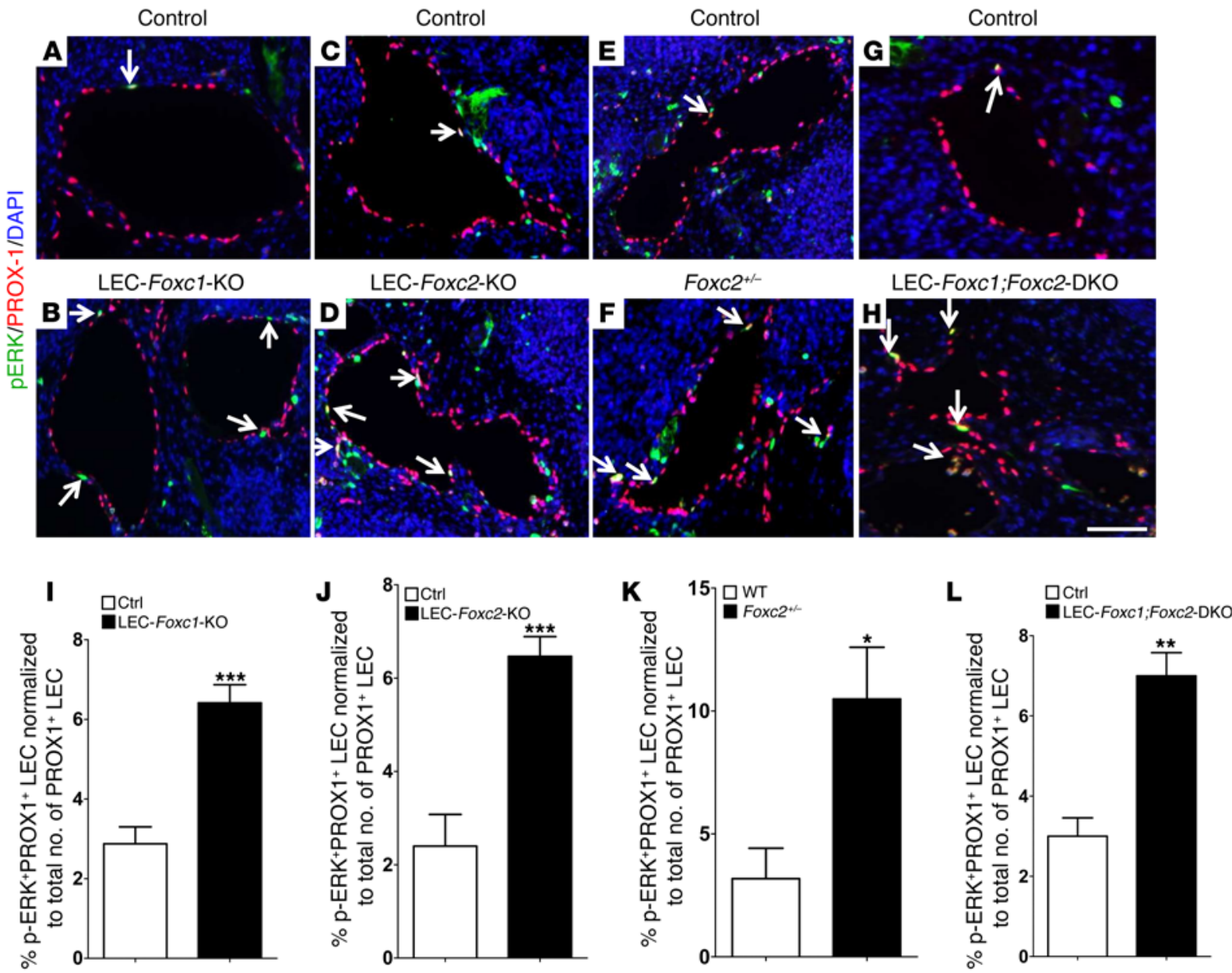

Figure 6. LEC-Foxc-KO mice display increased ERK activation in the lymph sacs. (A-H) p-ERK immunostaining of E12.5 lymph sacs. Arrows indicate p-ERK + PROX $1^{+}$LECs. Scale bar: $100 \mu \mathrm{m}$. (I-L) Quantitative analysis of PROX1 and p-ERK immunostaining from E12.5 lymph sacs showing an increase in the number of p-ERK ${ }^{+}$LECs in LEC-Foxc1-KO (I), LEC-Foxc2-KO (J), global-Foxc2+/- (K) and LEC-Foxc1; Foxc2-DKO (L) embryos. $P$ values were obtained by 2-tailed Student's $t$ test. Data are presented as mean \pm SEM. ${ }^{*} P<0.05,{ }^{* *} P<0.01,{ }^{* * *} P<0.001 . n=3$.

structurally, RASA1 has SH2 and SH3 domains, which recognize phosphotyrosine- and proline-rich sequences in intracellular signaling proteins, respectively, whereas RASA4 and RASAL3 do not. Each of the three has a pleckstrin homology $(\mathrm{PH})$ domain that binds membrane phospholipids. Although RASA4 is highly expressed in lymph nodes (56), whether these RasGAP proteins are involved in other lymphatic processes, including lymphatic valve formation, needs to be investigated fully in the future. Two ChIP+ FOXC2-binding sites have previously been identified in the locus of RASGRP3, a Ras activator, in human LECs (20), and consistent with this result, we found that FOXC2 was able to bind to RASGRP3-ECR1/2 and RASGRP3-ECR2 in HMVEC-dLyNeo and HUVECs, respectively (Supplemental Figure 5, E and F) and that RasGrp3 was significantly upregulated in Foxc1/c2-mutant LECs (Supplemental Figure 5D). FOXC1 and FOXC2 negatively regulate gene expression in other systems $(57,58)$, and FOXC's transcriptional activity of each downstream gene may be complex and dependent on cofactors and/or posttranslational modifications $(59,60)$.

The molecular interactions between FOXC1/C2 and the VEGF-C/VEGFR3/Ras pathway are likely to be reciprocal. Whereas the Ras/ERK cascade via the VEGF-C/VEGFR3 axis regulates VEGFR3 expression (4), VEGFR3 signaling cooperates with FOXC2 in lymphatic vessel development (21), and the loss of Foxc2 leads to increased VEGFR3 internalization in LECs, indicative of active VEGFR3 signaling (20). Overall, given evidence that most of the genes mutated in lymphatic malformations encode proteins involved in activation of the VEGFR3 signaling pathway (54), the molecular interaction between FOXC and Ras/ERK signaling will likely elucidate mechanisms underling the fine-tuning of VEGFR3 signaling in LECs. Given evidence that lymphatic vessels in individuals with inactivating mutations in FOXC2 are hyperplastic (17-19), one of the intriguing findings in this study is the rescue of the enlarged lymphatic vessels in LEC-Foxc2-KO embryos, as well as in LEC-Foxc1-KO and LEC-Foxc1;Foxc2-DKO embryos, by pharmacological inhibition of ERK activation (Figure 7 and Supplemental Figure 6). To our knowledge, this is the first report showing blockade of the lymphatic abnormalities caused by the loss of FOXC2. There is evidence that FOXC2 expression levels are significantly reduced in the duodenal mucosa in individuals with idiopathic intestinal lymphangiectasia, a pathologic dilation of lymph vessels (61), and that a heterozygous FOXC2 mutation causes pulmonary lymphangiectasia (62). Thus, our 
A

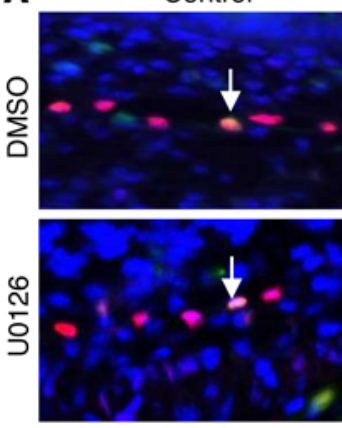

B
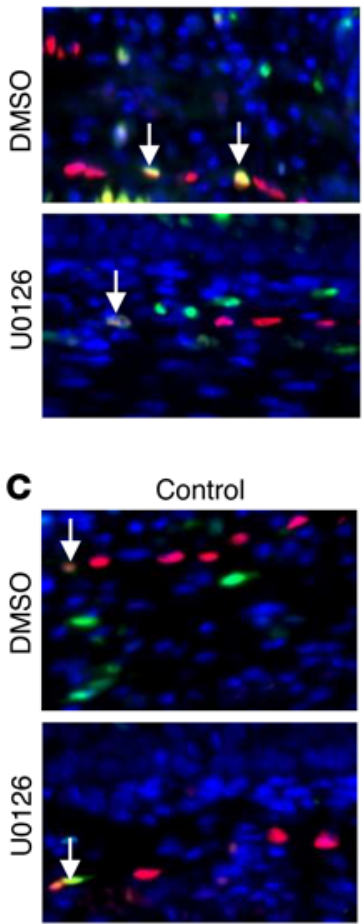
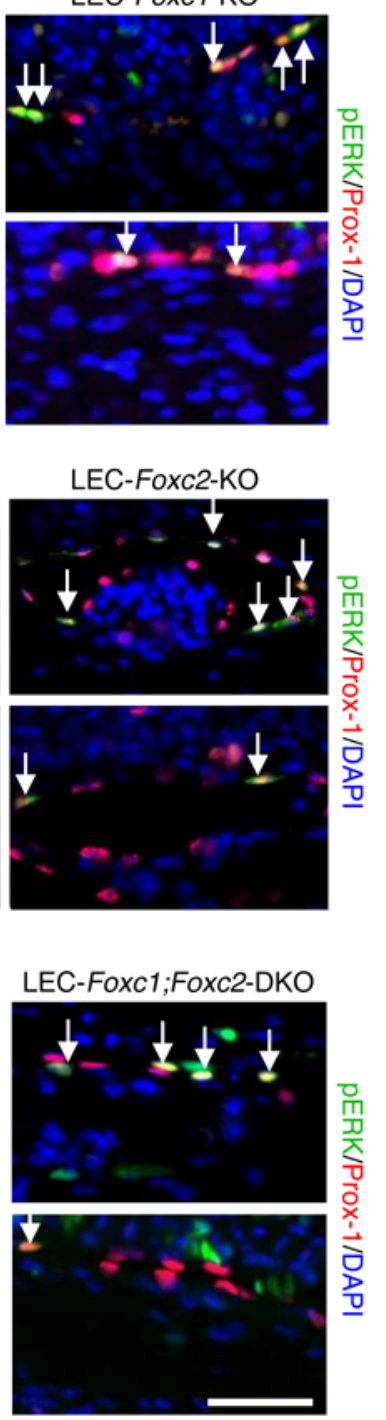

LEC-Foxc2-KO
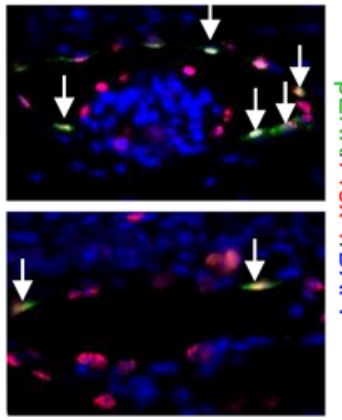

LEC-Foxc1;Foxc2-DKO
D
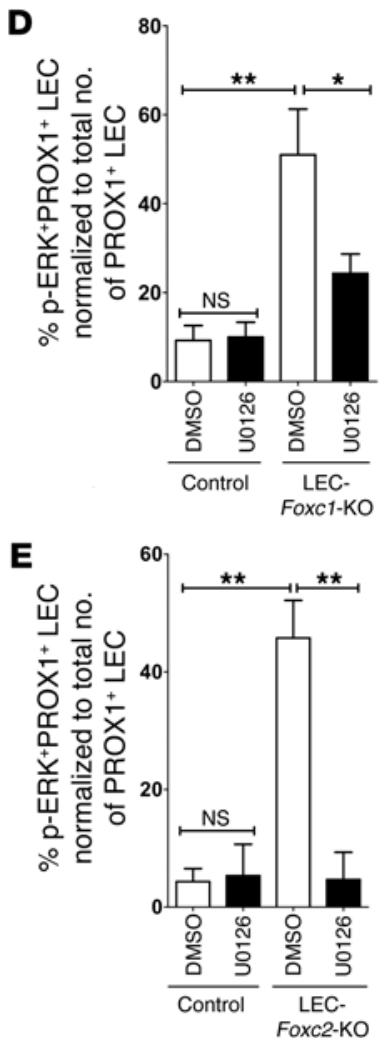

$\mathbf{F}$

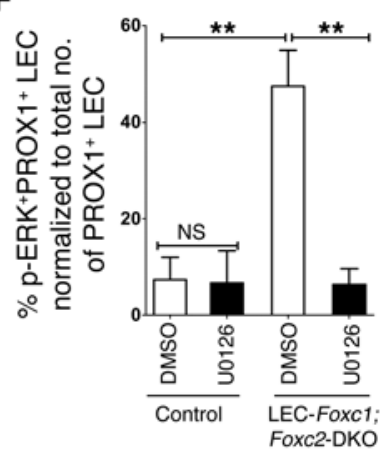

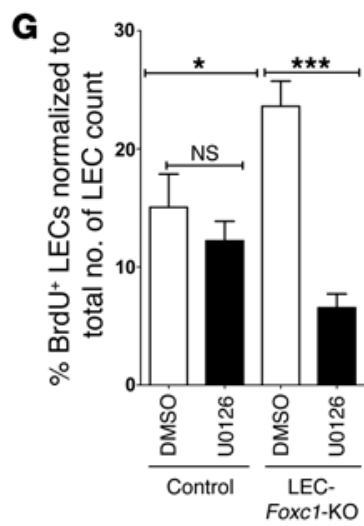

H
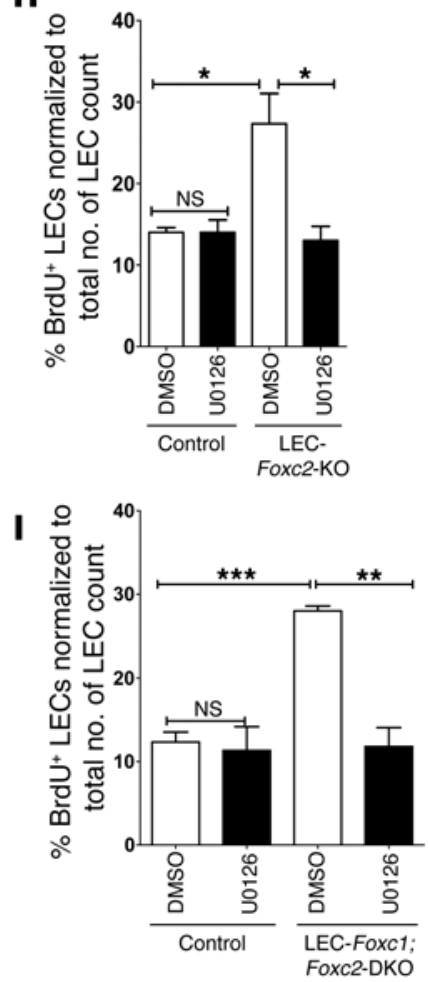

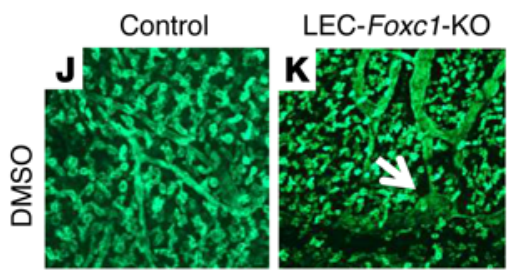

Control

LEC-Foxc2-KO

Control

LEC-Foxc1;Foxc2-DKO
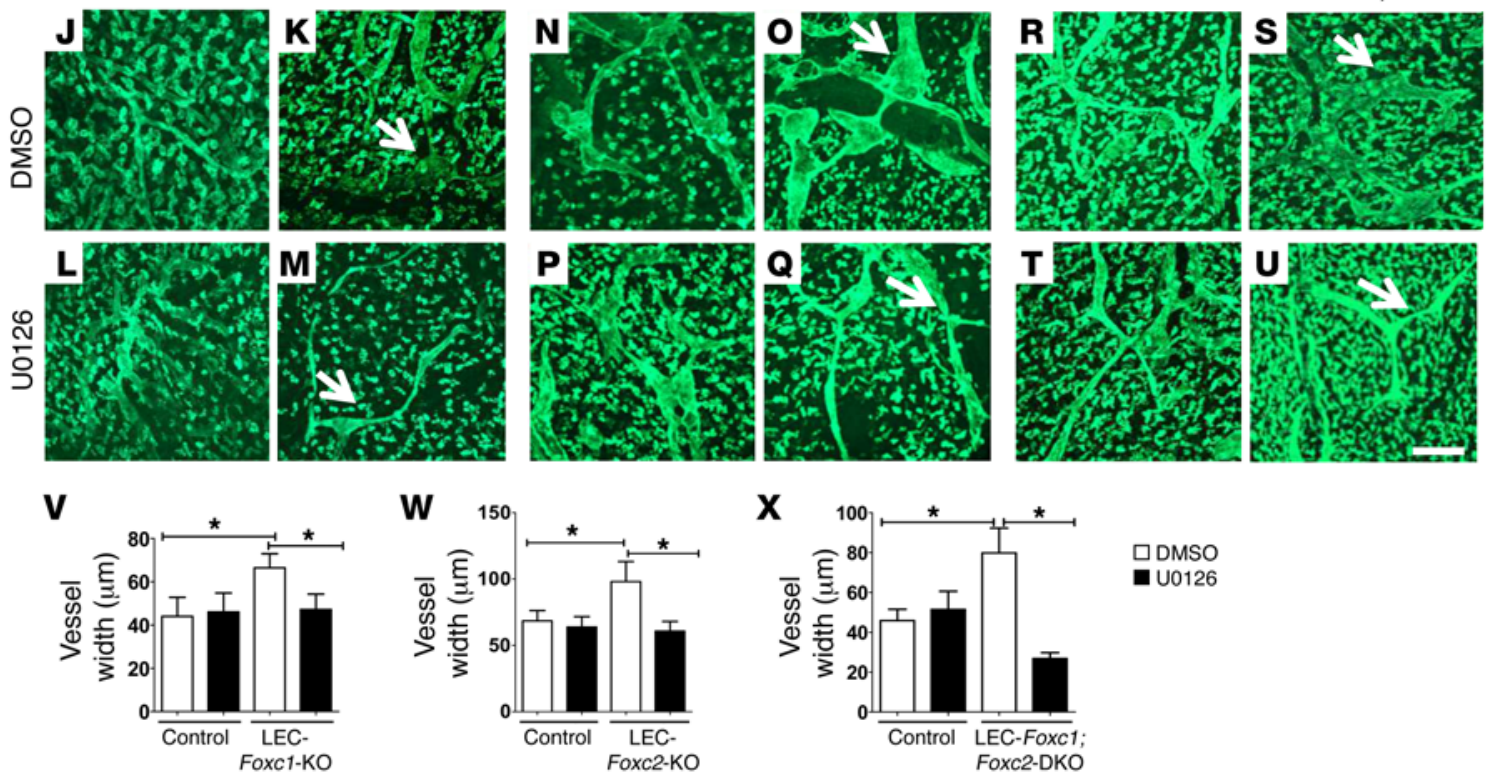
Figure 7. Inhibition of ERK hyperactivation in LEC-Foxc-KO mice rescues enlarged lymphatic vessels. (A-C) Formation of hyperactivated ERK ${ }^{+}$ LECs in LEC-Foxc-KO embryos is diminished by treatment with the MEK

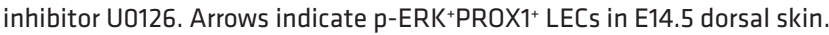
(D-F) Quantitative analysis of PROX1/p-ERK immunostaining of E14.5 dorsal skin showing an increase in the number of $p-E R K^{+}$LECs in LECFoxc-KO embryos, rescued by treatment with the MEK inhibitor U0126, in LEC-Foxc1-KO (D, $n=3)$, LEC-Foxc2-KO (E, $n=4)$, and LEC-Foxc1;Foxc2-DKO $(\mathbf{F}, n=3$ ) embryos. (G-I) Quantitative analysis of BrdU+LYVE-1+ LECs in E14.5 dorsal skin showing an increase in the number of proliferating LECs in LEC-FoxC-KO embryos, rescued by treatment with the MEK inhibitor U0126, in LEC-Foxc1-KO (G), LEC-Foxc2-KO (H), and LEC-Foxc1;Foxc2-DKO embryos (I). $n=4$. (J-X) LYVE-1 immunostaining of E15.5 dorsal skin (J-M, $\mathbf{N}-\mathbf{Q}$, and $\mathbf{R}-\mathbf{U}$ ) and morphometric analysis $(\mathbf{V}-\mathbf{X})$ showing the rescue of the lymphatic phenotype in all 3 lines of the LEC-Foxc-KO embryos by the MEK inhibitor U0126. $n=4$. $P$ values were obtained by 2 -tailed Student's $t$ test. Data are presented as mean \pm SEM. ${ }^{*} P<0.05$, ${ }^{* *} P<0.01,{ }^{* * *} P<0.001$. Scale bar: $50 \mu \mathrm{m}$

results may open up a new avenue of treatment for lymphatic anomalies and pathological lymphatic vessel remodeling associated with lymphangiogenesis.

Recent studies indicate that Ras/Raf/ERK signaling induces lymphatic cell fate specification (42). PROX1, COUP-TFII, and Notch signaling participate in a feedback loop that maintains the balance between venous and lymphatic cell fate in progenitors (6), and the lack of Notch1 increases the number of PROX1+ LECs in the cardinal vein and lymph sacs (63). Interestingly, we found that expression of Notch receptors and ligands such as Notch1 is downregulated in Foxc1/c2-mutant LECs, and that activation of Notch signaling is reduced in LEC-Foxc-KO embryos (Supplemental Figure 8). Therefore, besides LEC proliferation, it is plausible that FOXC1 and FOXC2 participate in early LEC specification by regulating the ERK and Notch pathways.

We show that FOXC1 is important for valve maturation (Supplemental Figure 7). FOXC2 deficiencies in mice and humans prevent the initial process of lymphatic valve generation (20, $21,45)$, but FOXC2 continues to be expressed in the valveforming cells throughout lymphatic maturation after formation of the valve territory $(20,22)$. Our RNA-seq analysis revealed that expression of key signaling genes in valve maturation and maintenance, including Sema3a, Plxna1, and Efnb2 (64-66), was significantly downregulated in Foxc1/c2-mutant LECs at E15.5 (Supplemental Table 3), and ChIP ${ }^{+}$FOXC2-binding sites in the Sema3a and Efnb2 loci have been identified in human LECs (20). It is tantalizing to consider that FOXC1 and FOXC2 may act upstream of the SEMA3A/PLXNA1 and EFNB2 signaling pathways during lymphatic valve maturation.

In conclusion, our findings identify a molecular interaction by which both FOXC1 and FOXC2 control lymphatic vessel growth. Our results not only increase basic scientific knowledge of lymphatic cell biology, but also are of high clinical significance for the pathology of lymphangiogenesis.

\section{Methods}

Mice. Foxc1 $1^{+/-}$(24), Foxc1 $1^{f / f l}$ (34), Foxc2 $2^{+/-}$(55), Foxc2 $2^{f / f l}$ (34), Prox1$\operatorname{CreER}^{T 2}$ (35) (a gift from Guillermo Oliver, St. Jude Children's Research Hospital, Memphis, Tennessee, USA), and Tie2-Cre (49) (a gift from
Rong Wang, UCSF, San Francisco, California, USA) mice were used. LEC-specific Foxc1, Foxc2, and compound Foxc1;Foxc2 mutant mice were generated for analysis by crossing $F o x c 1^{f / f l}, F o x c 2^{f / f l}$, and $F o x c 1^{f / f l}$ Foxc $2^{f / f l}$ females with Foxc1 $1^{f / f l}$ Prox1-CreER ${ }^{T 2}$ (LEC-Foxc1-KO), Foxc $2^{f / / f l}$ Prox1-CreER $R^{T 2}$ (LEC-Foxc2-KO), and Foxc1 $1^{f / f l}$ Foxc $2^{f / f l}$ Prox1-CreER $R^{T 2}$ (LEC-Foxc1;Foxc2-DKO) males, respectively. Foxc1/f/fl Tie2-Cre male mice were crossed with Foxc ${ }^{f / f l}$ female mice to generate EC-specific Tie2-Cre Foxc1 $1^{f / f l}$ (EC-Foxc1-KO) mice, as described previously (48). Embryonic age was determined by defining noon on the day of vaginal plug as E0.5. Tamoxifen ( $5 \mathrm{mg} / 40 \mathrm{~g}$ body weight) dissolved in corn oil was injected intraperitoneally into pregnant dams at E10.5. Genotyping of LEC-specific Foxc conditional mutants was performed by Transnetyx Inc. Control assessments were performed in the mutants' respective littermates $\left(F o x c 1^{f / f l}, F o x c 2^{f / f l}\right.$, or Foxc $\left.1^{f / f l} F o x c 2^{f / f l}\right)$, as we did not observe any adverse effects in either Prox1-CreER ${ }^{T 2}$ or Tie2-Cre embryos.

Immunohistochemical analyses. The lymphatic vasculature was analyzed by whole mount LYVE-1 immunostaining of the dorsal embryonic skin at E15.5. Embryos were harvested in cold PBS and fixed overnight in $4 \%$ paraformaldehyde (PFA) at $4^{\circ} \mathrm{C}$. Following fixation, the embryos were stored overnight in $100 \%$ methanol at $-20^{\circ} \mathrm{C}$. The skins were then obtained under a dissection microscope, rehydrated, and blocked in $10 \%$ donkey serum before staining with antiLYVE-1 antibody (Abcam, ab14917). For immunohistochemistry using cryosections, depending on the embryonic age, the embryos were fixed in 4\% PFA for 30-60 minutes, followed by overnight treatment with $30 \%$ sucrose solution at $4^{\circ} \mathrm{C}$. The embryos were then embedded in OCT solution and frozen in a LN/methanol bath. Cryosections (10 $\mu \mathrm{m}$ thick) were made the next day using a cryostat.

$\beta$-Gal immunostaining was performed using a combination of anti- $\beta$-gal (rabbit polyclonal, Cappel 55976)/PECAM-1 (rat monoclonal, clone 13.3, BD Biosciences, 553369)/PROX1 (R\&D Systems, AF2727) for E10.5 embryos (cardinal vein sections) and anti- $\beta$-gal/ LYVE-1 (rat monoclonal clone ALY7, MBL, D225-3)/PROX1 (R\&D Systems, AF2727) antibodies for E12.5 embryos (lymph sac sections).

For analysis of LEC proliferation at E12.5 and E14.5, pregnant dams received $150 \mathrm{mg} / \mathrm{kg}$ BrdU dissolved in DMSO 2 hours prior to dissection of embryos. The embryos were then fixed in $4 \%$ PFA, and 10 serial cryosections ( $10 \mu \mathrm{m}$ thick) of the lymph sacs through the neck (for E12.5 embryos) and dorsal skin (for E14.5 embryos) were stained with anti-BrdU (Abcam, ab6326) and anti-LYVE-1 (Abcam, ab14917) antibodies to detect proliferating LECs. Images were captured at 20x magnification. LYVE-1 ${ }^{+}$BrdU ${ }^{+}$LECs were manually counted throughout the lymph sacs or dorsal skin sections. The total number of $\mathrm{BrdU}^{+}$ LECs was then normalized against total number of LECs counted. Lower-magnification $(4 \times)$ images were also captured to highlight the difference in size of the control and mutant lymph sacs. Additionally, LEC apoptosis was analyzed in the dorsal skin at E15.5 and lymph sacs at E12.5 using a TUNEL assay kit (Roche). Briefly, 10 serial (10- $\mu \mathrm{m}$-thick) dorsal skin/lymph sac sections collected at the level of heart (for dorsal skin)/neck (for lymph sacs) were stained with anti-LYVE-1/TUNEL and anti-PROX1/TUNEL antibodies. The sections were subsequently counterstained with DAPI. Images of lymphatic vessels were captured at $20 \times$ and $40 \times$ magnifications. LYVE- $1^{+}$TUNEL $^{+}$DAPI $^{+}$LECs and PROX $1^{+} \mathrm{TUNEL}^{+}{ }^{+} \mathrm{DAPI}^{+}$LECs were manually counted and normalized to the total number of LECs counted.

p-ERK/PROX1 (R\&D Systems, AF2727) staining was analyzed in lymph sacs at E12.5 and dorsal skin at E14.5. Briefly, the embryos were 
fixed in 4\% PFA overnight and processed for paraffin embedding. Serial (10- $\mu \mathrm{m}$-thick) lymph sac/dorsal skin sections were collected at the level of neck (for lymph sacs)/heart (for dorsal skin) and stained for p-ERK (Cell Signaling Technology, 4370)/PROX1 (R\&D Systems, AF2727). The sections were subsequently counterstained for Hoechst nuclear stain. Images of lymph sacs/lymphatic vessel were captured at $10 \times$ magnification. $\mathrm{p}-\mathrm{ERK}{ }^{+} \mathrm{PROX} 1^{+} \mathrm{Hoech}^{+} \mathrm{LECs}$ from lymph sacs (E12.5) and dorsal skin (E14.5) were manually counted and normalized against total number of LECs counted. Only cells that had nuclear localization of p-ERK were included in the quantification analyses.

Cell junctions were analyzed in Foxc mutants by immunostaining of dorsal skin sections in E15.5 embryos with anti-CDH5 (R\&D Systems, AF1002)/LYVE-1 antibodies (Abcam, ab14917). Ten serial sections at the level of heart were analyzed, and images were captured at $63 \times$ magnification. All sections were counterstained with DAPI.

Whole mount mesentery staining was performed as follows. The mesentery tissue was harvested from embryos/pups using fine forceps. The tissue was then laid out in a plastic petri dish over ice and left until it was firmly attached to the base. Following fixation with $4 \%$ PFA, the tissue was stained for various antibodies, including FOXC1 (Cell Signaling Technology, 7415), FOXC2 (R\&D Systems, AF6989), and PROX1 (R\&D Systems, AF2727), at $4^{\circ} \mathrm{C}$.

Whole mount immunostaining was documented by Z-sectioning using a Zeiss UV-LSM-510 or an LSM-510 Meta confocal microscope; immunostaining on tissue sections was examined and recorded using a Zeiss AxioVision fluorescence microscope.

FACS. Cells isolated from E15.5 controls and conditional Foxcmutant (LEC-Foxc1-KO, LEC-Foxc2-KO, LEC-Foxc1;Foxc2-DKO) embryos were stained for either LYVE-1 (Abcam, ab14917)/CD31 (BD Pharmingen, 553373) or LYVE-1 (Abcam, ab14917)/PROX1 (R\&D Systems, AF2727) and subjected to FACS. Briefly, E15.5 embryos were harvested in HBSS (Sigma-Aldrich) and then chopped for an overnight digestion with collagenase I/II. The collagenase-treated cell suspension was then incubated with rbc lysis buffer (STEMCELL Technologies). Following centrifugation, cell pellets were incubated with antiLYVE-1 antibody (Abcam) for 20 minutes at $4^{\circ} \mathrm{C}$. After washing with PBS, the cells were stained with PE-conjugated anti-CD31 antibody (BD Pharmingen, 553373) and Alexa Fluor 488-conjugated donkey anti-rabbit secondary antibody (Invitrogen, A21206). For the LYVE-1/ PROX1 staining combination, following rbc lysis treatment the cells were fixed and permeabilized in ice-cold methanol at $-20^{\circ} \mathrm{C}$ for 10 minutes. The cells were then washed 3 times in $0.01 \%$ PBST. The rest of the staining protocol was followed as stated above.

After gauze filtration with a cell strainer $(40 \mu \mathrm{m}$; BD Biosciences $)$ to obtain a single-cell suspension, the cells were directly sorted into RNA protect solution (QIAGEN) to acquire a pure LEC population on a MoFlo cell sorter (Beckman Coulter).

For additional apoptosis analysis of the total LEC population in Foxc mutants, the LECs were isolated from E15.5 embryos as explained above. Following the staining with LYVE-1/CD31 antibodies, the cells were further stained with Annexin V-APC conjugate (BD Pharmingen, 550474). Cell death was then analyzed on a BD Fortessa flow cytometer.

Culture of human LECs. Human primary dermal LECs (HLEC-1, HLEC-2, HLEC-3) were isolated from neonatal foreskins and cultured as described by Choi et al (67). HMVEC-dLyNeo (Lonza) were cultured and maintained according to the manufacturer's protocol.
RNA extraction and qPCR. RNA was extracted from sorted LECs using an RNeasy Mini Kit (QIAGEN) following the manufacturer's protocol. The HLECs and HUVECs were subjected to total RNA extraction using RNAstat solution (Tel-Test), again following the manufacturer's protocols. The extracted RNA was subjected to DNase treatment to disintegrate the genomic DNA. The concentration of RNA was determined using a NanoDrop machine (Thermo Scientific). cDNA was synthesized with an iScript reverse transcriptase kit (Bio-Rad) in accordance with the manufacturer's instructions. Quantitative PCR (qPCR) was performed using a Fast qPCR machine (Applied Biosystems), Fast SYBR reaction master mix (Applied Biosciences), TaqMan Universal Master Mix (Applied Biosystems), and gene-specific primer sets. Peptidylprolyl isomerase A (Ppia) or $G A P D H$ was used as an internal standard for mRNA expression for mouse or human samples, respectively. Primer sequences are provided in Supplemental Table 4.

Measurement of $L V W$. To measure LVW, we created new software using MATLAB (MathWorks) based on the same fundamental algorithm for distance determination as in Image (NIH). However, we built in batch image processing, which allows the user to queue a number of images for analysis by designating a directory in which they are located. It is a more streamlined method of LVW measurement, cutting down on the time required for analysis. We found no difference between LVWs determined using our MATLAB-generated software and LVWs determined by conventional methods (37).

RNA-seq. RNA-seq was performed using RNA extracted from sorted LYVE-1 $1^{+}$CD $31^{+}$LECs isolated from E15.5 LEC-Foxc1;Foxc2DKO and littermate controls at the University of Chicago Genomics Core. LECs from several embryos were pooled together for respective mutants and controls. Two pools of LECs isolated from control embryos wherein each pool had cells from 2-3 embryos were used for RNA extractions. Similarly, 2 pools of LECs isolated from LECFoxc1;Foxc2-DKO embryos wherein each pool had cells from at least 2 embryos were used. A 2-lane 50-bp single-end RNA-seq was performed according to a protocol on an Illumina HiSeq2500 instrument. Briefly, RNA quality was checked by determining the RNA integrity number (RIN) on a bioanalyzer (Agilent Technologies). All the RNA samples for the sequencing experiments had RIN $>9$. Libraries from the above RNA samples were created using a Ribo-Zero depletion kit (Illumina). Each sample (biological replicates) was then given a unique ID barcode during this process. Last, the pooled samples (biological replicates) were sequenced and stored in FASTQ format. The data were then analyzed at the Northwestern University Next Generation Sequencing (NGS) Core using Cufflinks software (http://cole-trapnelllab.github.io/cufflinks/) to test the differential regulation of genes between mutants and control embryos. During analysis one pool of control LECs was eliminated and considered an outlier based on clustering in comparison to other samples.

Administration of U0126 and PD0325901 for inhibition of ERK in mice. Following timed mating, mice with confirmed vaginal plug were injected with either U0126 (Promega; $5 \mathrm{mg} / \mathrm{kg}$ body weight) or an equivalent amount of DMSO dissolved in PBS at E12.5 and E13.5. Following the injections, embryos were harvested at E14.5 and E15.5 to study the effects of U0126 on lymphatic vessel formation. PD0325901 was dissolved in DMSO (Sigma-Aldrich, $3 \mathrm{mg} / \mathrm{kg}$ body weight) and injected in pregnant dams at E12.5. DMSO-injected dams were used as controls. 
Forkhead transcription factor binding prediction and sequence analysis. Putative FOXC-binding sites were determined first by searching for evolutionarily conserved regions within each suspect gene using the ECR Browser (68) to compare human and mouse genomes. Sequences for conserved regions of both species were then examined in ScanProsite (69) using general FOXC-binding motifs ACAAA, ATAAA, AAACA, AAATA, TGTTT, TTTGT, TTTAT, and TATTT. Sequences adjacent to any detected motifs were further analyzed for elements characteristic of FOXC-binding sites, such as high adenine (A) content (20). Suspected FOXC-binding site regions were aligned and compared using the ECR Browser to ensure that the regions are highly conserved and that the binding elements are present in both genomes. Conserved FOXC-binding elements were then fed into the UCSC Genome Browser (70) to obtain sequences (as shown below) with 500-bp overhangs on both the $5^{\prime}$ and $3^{\prime}$ ends to allow for ChIP primers to be designed across these regions. Additionally, hypersensitivity regions that indicate active chromatin were determined for all the putative binding sites as per work reported and summarized on the Encyclopedia of DNA Elements (ENCODE; https://genome.ucsc. edu/ENCODE/), using the UCSC Human Genome Browser:

RASA4-ECR 6. >hg19_dna range $=$ chr7:102249782-102250928 $5^{\prime}$ pad=500 $3^{\prime}$ pad=500 strand=+ repeatMasking=none; CGGCTCACTGCAACCTCTGCCTCCCAGGTTCAAGCAATTCTCCCTGCCTC AGCTTCCTGAGTAGCTGGGATTACAGACGCCTGCCACCACGC CCAGCTAATTTTGTCATTTTCGTAGAGACAGGGTTTCACCCTGTTGGCCAGGCTGCTCTCGAACTCCTGACCTCAGGTGATCTGCCCACCTCGGACTTCCAAAGTGCCGGGATGACAGGCGTGAGCCACCGTGCCCAGCCTCTCCAGCTGTTTTACATTTGGGGAAACTGAGATCACAGTTGGCAGGCATTGGCCTCAGGTCACAGGGTAGCTGCCTCAGGGCGACTGGTGAGCCTGGCCTCACATCCTCACCCAGCT.

RASA4-ECR 10. >hg19_dna range $=$ chr7:102244948-102245956 $5^{\prime}$ pad=500 3'pad=500 strand=+ repeatMasking=none; GAGCGACTTGGGGAGGTGGCTTTGGAGCCGGAAGGGAAGACGCTTCTCACGGACAGAGTGTGACTCAGACCCAGGCCCAGACGGGAG TGCAGCCTGCAGCTAAAAATACCCTGGGGGTTGGGGGGTG GCCCTGTCACAGGTGACTCAGGCCCATGGAACCATCCAAACCACTGTCCCTCCTTGGGGGAACCCTCAATTTGCCCATCGGGAGATTTTCAGCAACTCTTTTCACCCCCCCAGCCCCACGAGG.

RASA4-ECR 11. >hg19_dna range $=\mathrm{chr7}: 102243818-102244828$ $5^{\prime}$ pad=500 $3^{\prime}$ pad=500 strand=+ repeatMasking=none; GCAGAGGTTGCAGTGAGCCAAGAAAACGCCACTGCACTCCAGCCTGGGCGACAGAGCCAGACTGCCTCAAAAAAAAAAAAAAAAAAAAAACCACCAAAAAAAAAAAAACAGCCCGGGTGCAGTGGCTCACG CCTGTAATCCCAGTACTTTGGGAGGCCGAGGCAGGCAGATCACCTGAGGTCAGGAGTTTGAGACCAGCCTAGCCAACATAGTGAAGTCCCGTCTCTACTAAAAATACAAAAAATTAGCC.

RASA4-ECR 17. >hg19_dna range=chr7:102239289-102240298 $5^{\prime}$ pad=500 3' pad=500 strand=+ repeatMasking=none; AAGATTGTGCCGCTGCACTCCAGCCTGGGTGACAGAGCAAGACTCCATCTCAAAAAAAAGACAGAAGACAGAGCTGAGCTGAATGCAGAACTGTGGGGAAAAATAAAGGGGAGGGGCCGGGCGCGGTGGCTCATGCCTGTAATCCCAGTACTTGGGGAGGCCGAGGCGGGTGGATCACAAGGTCAGGAGATCGAGACCATCCTGGCTAACATGGTGAAACCCTGTCTCTACTAAAAATGCAAAAAATTAG.

RASAL3-ECR 2. >hg19_dna range=chr19:15574278-15575287 $5^{\prime}$ pad=500 3' pad=500 strand=+ repeatMasking=none; GGGGGAA-
AGAGGGAACAGGGTAGCTGGGCCCCAGCGGCTTCCTGCTG GGCTTTTGCACTCCCCACAACTTCCTGTGTCTGTATGGTTG ATGCTCCGGAGGCAGACAAAACCCAAGCATCCGGCTTCCCAGCCCGGCCCAAGCTCAGGGTGGGGGAACCATGTACCTGGGGCCTCCTGCTCCGGCTCCGGGTCTGGCTCCGGCGGTGGGTTCTTATGCCTCCCCCAGAGGGCCTTGGAGAGTCGAAGGCGA.

RASAL3-ECR 5. >hg19_dna range $=$ chr19:15572246-15573253 $5^{\prime}$ pad=500 $3^{\prime}$ pad=500 strand=+ repeatMasking=none; TACCACTCCACACACCTTCTTGCATTGAATCCTCCAAAAGCCATTCAAA GGGCTATGATAACAGGCTCAGAGAGGTTGAGTGACTTGCTC AGGGTCACACAGTTTGTGAGTGGTTGGGCTGGGCTTCAGTC ACAAGCATTCTTCTCTCTCTCTCTTTTTTTGTTTTTTTGTTT TTTTTGCATTCTTCTCTTAACCAAGACATTATGTGGGTCCAAGAGGTTAAAAAACAGGACAGGGGCTGGGCAAGGTG.

RASAL3-ECR 13. >hg19_dna range $=$ chr19:15566879-15567889 $5^{\prime}$ pad=500 3' pad=500 strand=+ repeatMasking=none; GCAGGCCGCTGACATGGACTCTCCCCCAGCTCACCCAGGGTCTCCTGG AGGTAATCCTGTGCCACGAGCTTCATGTACTCATCGATAGCCT TGGTGGCCAATGTGTTTTCCCGGAACAGCAGCGCCTCACGG CCTCCACAGCGCGCCAGCTCCGCAGTGCCCAGGTCAGTCACCAGCGCCTAGGAAGGGCAGGAGGTCAGGTTGCACCAGATGCAGACAGAGTCCAAGGGAATTCGGATCCTTGGCTGGT.

RASAL3-ECR 17. >hg19_dna range=chr19:15563076-15564084 $5^{\prime}$ pad=500 3' pad=500 strand=+ repeatMasking=none; GGAGCCTG GAGTCCAGATCCTGCAGCTGGCCCCGCAGCTGCTCCTGCTG CTCCGTCAAGGCCCGGATTTGGGTGCTCAGCGACTCCACGA GGCGGGACAGCACTTTCTGCTCCTCACGCAGAGCGGCCAC CTCGCACTGCAGCTCTGCCAACTGTGGTGGGAGAGCAGCTGTAATCTGACCCGTTGTCCCGCCCCTGTGTCTCCCCGGAGACCCTGACCTCCCAGGCCCAAGCGCTCAGCGTCATCACCG.

ChIP assay. Confluent LECs (HMVEC-dLyNeo) and HUVECs (Lonza) were purchased and cultured in EBM medium with the EGMMV BulletKit (Lonza). The cells were cross-linked with $1 \%$ formaldehyde, followed by sonication. The sheared chromatin was immunoprecipitated with anti-FOXC2 antibodies (Santa Cruz Biotechnology Inc., sc-31734x and Abcam, ab5060), anti-FOXC1 antibodies (Origene, TA302875 and Abcam, ab5079), or control IgG and protein G Sepharose beads. Heating of the immunoprecipitates at $65^{\circ} \mathrm{C}$ for 6 hours was carried out to reverse formaldehyde cross-linking. DNA was extracted from eluted immunoprecipitates using a QIAGEN PCR purification kit and subjected to PCR with specific primers. Input lysates were collected before immunoprecipitation. ChIP primer sequences are provided in Supplemental Table 5.

Knockdown of FOXC2 and measurement of Ras activation. HUVECs from pooled donors (Lonza) were cultured (passages 2-7) on 0.1\% gelatin-coated plates in EndoGRO-LS complete culture medium (EMD Millipore). HUVECs were grown to confluence in 6-well plates and transfected with a nonspecific, negative control siRNA (QIAGEN) or siRNA targeted toward FOXC2 (5'-AGGUGGUGAUCAAGAGCGAUU-3', GE Dharmacon) at a final concentration of $33 \mathrm{nM}$ using Lipofectamine RNAiMAX Reagent (Invitrogen) for 6 hours according to the manufacturer's instructions. At that time medium was replaced with EndoGRO-LS complete culture media, and cells were allowed to recover for 72 hours. Transfected HUVECs were then serum deprived overnight in EndoGRO basal medium and stimulated with VEGF (50 $\mathrm{ng} / \mathrm{ml}$ ) for 10 minutes where indicated. Cell cultures were lysed in 
1× RIPA buffer (Cell Signaling Technology) containing protease (Cell Signaling Technology) and phosphatase (Roche) inhibitors, $100 \mu \mathrm{l}$ per 3 wells of a 6-well plate. Lysates were clarified by centrifugation at $14,000 \mathrm{~g}$ for 10 minutes at $4^{\circ} \mathrm{C}$, and the total protein concentration of supernatant was determined using a Pierce BCA protein assay kit (Thermo Scientific) according to the manufacturer's instructions. An equal amount of total protein for each experimental condition was loaded onto spin columns using an Active Ras Detection Kit (Cell Signaling Technology) according to the manufacturer's instructions, and bound protein was eluted using $2 \times$ Laemmli sample buffer and run on a $4 \%-20 \%$ SDS-PAGE gel. Samples were transferred to nitrocellulose and Western blotted with the Ras monoclonal antibody supplied with the Active Ras Detection Kit.

Statistics. Statistical analysis was performed using GraphPad Prism 5.0 (GraphPad Software). $P$ values were obtained by 2 -tailed Student's $t$ test. Data are presented as mean \pm SEM of representative experiments. A $P$ value less than 0.05 was considered significant.

Study approval. All procedures and animal studies were approved by Northwestern University's IACUC. Isolation of human LECs, provided by Young K. Hong, was approved by the University of Southern California Institutional Review Board.

\section{Author contributions}

$\mathrm{AF}$ and TK designed and analyzed the experiments. AF, YW, YU, and $\mathrm{PN}$ conducted the experiments. AF, AC, and FC provided
computational/MATLAB support for vessel width analysis. TL provided technical support for histology and immunostaining experiments. WHD provided technical support for mouse maintenance. MM provided technical help for cell culture. YM provided assistance in designing experiments and critically reviewed the manuscript. AF, PN, and TK wrote the manuscript.

\section{Acknowledgments}

We thank Tatiana Petrova, Susan Quaggin, Olga Volpert, and Ordan Lehmann for critical reading of the manuscript. We thank Guillermo Oliver and Rong Wang for providing the Prox1-CreER ${ }^{T 2}$ and Tie2-Cre mouse lines, respectively. We thank Yong K. Hong for providing human LECs. Immunofluorescence staining for p-ERK/ PROX1 was performed at the Northwestern University Research Histology and Phenotyping Laboratory, which is supported by National Cancer Institute (NCI) P30-CA060553 awarded to the Robert H. Lurie Comprehensive Cancer Center. This work was supported by the NIH (HL 126920 and EY019484 to T. Kume) and the American Heart Association (AHA-14POST20390029 to Y. Wang).

Address correspondence to: Tsutomu Kume, Feinberg Cardiovascular Research Institute, Department of Medicine, Northwestern University School of Medicine, 300 E Superior Street, Chicago, Illinois 60611, USA. Phone: 312.503.0623; E-mail: t-kume@ northwestern.edu.
1. Zheng W, Aspelund A, Alitalo K. Lymphangiogenic factors, mechanisms, and applications. J Clin Invest. 2014;124(3):878-887.

2. Karaman S, Detmar M. Mechanisms of lymphatic metastasis. J Clin Invest. 2014;124(3):922-928.

3. Stacker SA, Williams SP, Karnezis T, Shayan R, Fox SB, Achen MG. Lymphangiogenesis and lymphatic vessel remodelling in cancer. Nat Rev Cancer. 2014;14(3):159-172.

4. Brouillard P, Boon L, Vikkula M. Genetics of lymphatic anomalies. J Clin Invest. 2014;124(3):898-904.

5. Sevick-Muraca EM, King PD. Lymphatic vessel abnormalities arising from disorders of Ras signal transduction. Trends Cardiovasc Med. 2014;24(3):121-127.

6. Yang Y, Oliver G. Development of the mammalian lymphatic vasculature. J Clin Invest. 2014;124(3):888-897.

7. Francois M, et al. Sox18 induces development of the lymphatic vasculature in mice. Nature. 2008;456(7222):643-647.

8. Srinivasan RS, et al. The nuclear hormone receptor Coup-TFII is required for the initiation and early maintenance of Prox1 expression in lymphatic endothelial cells. Genes Dev. 2010;24(7):696-707.

9. Wigle JT, Oliver G. Prox1 function is required for the development of the murine lymphatic system. Cell.1999;98(6):769-778.

10. Karkkainen MJ, et al. Vascular endothelial growth factor $\mathrm{C}$ is required for sprouting of the first lymphatic vessels from embryonic veins. Nat Immunol. 2004;5(1):74-80.

11. Schulte-Merker S, Sabine A, Petrova TV. Lymphatic vascular morphogenesis in devel- opment, physiology, and disease. J Cell Biol. 2011;193(4):607-618.

12. De Val S, Black BL. Transcriptional control of endothelial cell development. Dev Cell. 2009;16(2):180-195.

13. Kume T. The cooperative roles of Foxc1 and Foxc2 in cardiovascular development. In: Maiese K, ed. Forkhead Transcription Factors: Vital Elements in Biology and Medicine. 2009;63-77.

14. Lam EW, Brosens JJ, Gomes AR, Koo CY. Forkhead box proteins: tuning forks for transcriptional harmony. Nat Rev Cancer. 2013;13(7):482-495.

15. Fang J, et al. Mutations in FOXC2 (MFH-1), a forkhead family transcription factor, are responsible for the hereditary lymphedema-distichiasis syndrome. Am J Hum Genet. 2000;67(6):1382-1388.

16. Mellor RH, et al. Mutations in FOXC2 are strongly associated with primary valve failure in veins of the lower limb. Circulation. 2007;115(14):1912-1920.

17. Brice $G$, et al. Analysis of the phenotypic abnormalities in lymphoedema-distichiasis syndrome in 74 patients with FOXC2 mutations or linkage to 16q24. JMed Genet. 2002;39(7):478-483.

18. Connell FC, et al. The classification and diagnostic algorithm for primary lymphatic dysplasia: an update from 2010 to include molecular findings. Clin Genet. 2013;84(4):303-314.

19. Mansour S, Brice GW, Jeffery S, et al. Lymphedema-distichiasis syndrome. In: Pagon RA, Adam MP, Ardinger HH, et al., eds. GeneReviews. Seattle, Washington, USA: University of Washington, Seattle; 1993-2016. https://www.ncbi.nlm.nih. gov/books/NBK1457/. Posted March 29, 2005. Updated May 24, 2012. Accessed April 25, 2016. 20. Norrmen C, et al. FOXC2 controls formation and maturation of lymphatic collecting vessels through cooperation with NFATc1. J Cell Biol. 2009;185(3):439-457.

21. Petrova TV, et al. Defective valves and abnormal mural cell recruitment underlie lymphatic vascular failure in lymphedema distichiasis. Nat Med. 2004;10(9):974-981.

22. Sabine A, et al. Mechanotransduction, PROX1, and FOXC2 cooperate to control connexin 37 and calcineurin during lymphatic-valve formation. Dev Cell. 2012;22(2):430-445.

23. Sabine A, et al. FOXC2 and fluid shear stress stabilize postnatal lymphatic vasculature. J Clin Invest. 2015;125(10):3861-3877.

24. Kume T, Deng KY, Winfrey V, Gould DB, Walter MA, Hogan BL. The forkhead/winged helix gene Mf1 is disrupted in the pleiotropic mouse mutation congenital hydrocephalus. Cell. 1998;93(6):985-996.

25. Lehmann OJ, et al. Novel anterior segment phenotypes resulting from forkhead gene alterations: evidence for cross-species conservation of function. Invest Ophthalmol Vis Sci. 2003;44(6):2627-2633.

26. Mears AJ, et al. Mutations of the forkhead/ winged-helix gene, FKHL7, in patients with Axenfeld-Rieger anomaly. Am J Hum Genet. 1998;63(5):1316-1328.

27. Nishimura DY, et al. The forkhead transcription factor gene FKHL7 is responsible for glaucoma phenotypes which map to 6p25. Nat Genet. 1998;19(2):140-147.

28. Smith RS, et al. Haploinsufficiency of the transcription factors FOXC1 and FOXC2 results in aberrant ocular development. Hum Mol Genet. 2000;9(7):1021-1032. 
29. French CR, et al. Mutation of FOXC1 and PITX2 induces cerebral small-vessel disease. J Clin Invest. 2014;124(11):4877-4881.

30. Ito YA, Walter MA. Genomics and anterior segment dysgenesis: a review. Clin Experiment Ophthalmol. 2014;42(1):13-24.

31. Dagenais SL, Hartsough RL, Erickson RP, Witte $\mathrm{MH}$, Butler MG, Glover TW. Foxc2 is expressed in developing lymphatic vessels and other tissues associated with lymphedema-distichiasis syndrome. Gene Expr Patterns. 2004;4(6):611-619.

32. Seo S, Fujita H, Nakano A, Kang M, Duarte A, Kume T. The forkhead transcription factors, Foxc1 and Foxc2, are required for arterial specification and lymphatic sprouting during vascular development. Dev Biol. 2006;294(2):458-470.

33. Amatschek S, et al. Blood and lymphatic endothelial cell-specific differentiation programs are stringently controlled by the tissue environment. Blood. 2007;109(11):4777-4785.

34. Sasman A, et al. Generation of conditional alleles for Foxc1 and Foxc2 in mice. Genesis. 2012;50(10):766-774.

35. Srinivasan RS, et al. Lineage tracing demonstrates the venous origin of the mammalian lymphatic vasculature. Genes Dev. 2007;21(19):2422-2432.

36. Yang Y, et al. Lymphatic endothelial progenitors bud from the cardinal vein and intersomitic vessels in mammalian embryos. Blood. 2012;120(11):2340-2348.

37. Fatima A, et al. Murine Notch1 is required for lymphatic vascular morphogenesis during development. Dev Dyn. 2014;243(7):957-964.

38. Baluk P, et al. Functionally specialized junctions between endothelial cells of lymphatic vessels. J Exp Med. 2007;204(10):2349-2362.

39. Ichise T, Yoshida N, Ichise H. H-, N- and Kras cooperatively regulate lymphatic vessel growth by modulating VEGFR3 expression in lymphatic endothelial cells in mice. Development. 2010;137(6):1003-1013.

40. Lapinski PE, et al. RASA1 maintains the lymphatic vasculature in a quiescent functional state in mice. J Clin Invest. 2012;122(2):733-747.

41. Pierrou S, Hellqvist M, Samuelsson L, Enerback S, Carlsson P. Cloning and characterization of seven human forkhead proteins: binding site specificity and DNA bending. EMBO J. 1994;13(20):5002-5012.

42. Deng Y, Atri D, Eichmann A, Simons M. Endothelial ERK signaling controls lymphatic fate specification. J Clin Invest. 2013;123(3):1202-1215.

43. Wu X, et al. MEK-ERK pathway modulation ameliorates disease phenotypes in a mouse model of Noonan syndrome associated with the Raf1(L613V) mutation. J Clin Invest.
2011;121(3):1009-1025.

44. Bazigou E, Makinen T. Flow control in our vessels: vascular valves make sure there is no way back. Cell Mol Life Sci. 2013;70(6):1055-1066.

45. Sabine A, Petrova TV. Interplay of mechanotransduction, FOXC2, connexins, and calcineurin signaling in lymphatic valve formation. Adv Anat Embryol Cell Biol. 2014;214:67-80.

46. Murtomaki A, et al. Notch signaling functions in lymphatic valve formation. Development. 2014;141(12):2446-2451.

47. Hayashi H, Kume T. Forkhead transcription factors regulate expression of the chemokine receptor CXCR4 in endothelial cells and CXCL12-induced cell migration. Biochem Biophys Res Commun. 2008;367(3):584-589.

48. Seo S, et al. Forkhead box transcription factor FoxC1 preserves corneal transparency by regulating vascular growth. Proc Natl Acad Sci US A. 2012;109(6):2015-2020.

49. Braren R, Hu H, Kim YH, Beggs HE, Reichardt LF, Wang R. Endothelial FAK is essential for vascular network stability, cell survival, and lamellipodial formation. J Cell Biol. 2006;172(1):151-162.

50. Bazigou $\mathrm{E}$, et al. Integrin-alpha9 is required for fibronectin matrix assembly during lymphatic valve morphogenesis. Dev Cell. 2009;17(2):175-186.

51. Koni PA, Joshi SK, Temann UA, Olson D, Burkly L, Flavell RA. Conditional vascular cell adhesion molecule 1 deletion in mice: impaired lymphocyte migration to bone marrow. JExp Med. 2001;193(6):741-754

52. Levet $\mathrm{S}$, et al. Bone morphogenetic protein 9 (BMP9) controls lymphatic vessel maturation and valve formation. Blood. 2013;122(4):598-607.

53. Winnier GE, et al. Roles for the winged helix transcription factors MF1 and MFH1 in cardiovascular development revealed by nonallelic noncomplementation of null alleles. Dev Biol. 1999;213(2):418-431.

54. Mendola A, et al. Mutations in the VEGFR3 signaling pathway explain $36 \%$ of familial lymphedema. Mol Syndromol. 2013;4(6):257-266.

55. Kume T, Jiang H, Topczewska JM, Hogan BL. The murine winged helix transcription factors, Foxc1 and Foxc2, are both required for cardiovascular development and somitogenesis. Genes Dev. 2001;15(18):2470-2482.

56. Zhang J, Guo J, Dzhagalov I, He YW. An essential function for the calcium-promoted Ras inactivator in Fcgamma receptor-mediated phagocytosis. Nat Immunol. 2005;6(9):911-919.

57. Mortazavi F, An J, Dubinett S, Rettig M.p120catenin is transcriptionally downregulated by FOXC2 in non-small cell lung cancer cells. Mol Cancer Res. 2010;8(5):762-774.
58. Silla ZT, Naidoo J, Kidson SH, Sommer P. Signals from the lens and Foxc1 regulate the expression of key genes during the onset of corneal endothelial development. Exp Cell Res. 2014;322(2):381-388.

59. Danciu TE, Chupreta S, Cruz O, Fox JE, Whitman M, Iniguez-Lluhi JA. Small ubiquitin-like modifier (SUMO) modification mediates function of the inhibitory domains of developmental regulators FOXC1 and FOXC2. J Biol Chem. 2012;287(22):18318-18329.

60. Ivanov KI, et al. Phosphorylation regulates FOXC2mediated transcription in lymphatic endothelial cells. Mol Cell Biol. 2013;33(19):3749-3761.

61. Hokari R, et al. Changes in regulatory molecules for lymphangiogenesis in intestinal lymphangiectasia with enteric protein loss. J Gastroenterol Hepatol. 2008;23(7 pt 2):e88-95.

62. de Bruyn G, et al. Hydrops fetalis and pulmonary lymphangiectasia due to FOXC2 mutation: an autosomal dominant hereditary lymphedema syndrome with variable expression. Eur J Pediatr. 2012;171(3):447-450.

63. Murtomaki A, et al. Notch1 functions as a negative regulator of lymphatic endothelial cell differentiation in the venous endothelium. Development. 2013;140(11):2365-2376.

64. Bouvree K, et al. Semaphorin3A, Neuropilin-1, and PlexinA1 are required for lymphatic valve formation. Circ Res. 2012;111(4):437-445.

65. Jurisic G, et al. An Unexpected role of semaphorin3a-neuropilin-1 signaling in lymphatic vessel maturation and valve formation. Circ Res. 2012;111(4):426-436.

66. Makinen T, et al. PDZ interaction site in ephrinB2 is required for the remodeling of lymphatic vasculature. Genes Dev. 2005;19(3):397-410.

67. Choi I, et al. Visualization of lymphatic vessels by Prox1-promoter directed GFP reporter in a bacterial artificial chromosome-based transgenic mouse. Blood. 2011;117(1):362-365.

68. Ovcharenko I, Nobrega MA, Loots GG, Stubbs L. ECR Browser: a tool for visualizing and accessing data from comparisons of multiple vertebrate genomes. Nucleic Acids Res. 2004; 32 (Web Server issue):W280-W286.

69. de Castro E, et al. ScanProsite: detection of PROSITE signature matches and ProRuleassociated functional and structural residues in proteins. Nucleic Acids Res. 2006;34(Web Server issue):W362-W365.

70. Miga KH, Newton Y, Jain M, Altemose N, Willard HF, Kent WJ. Centromere reference models for human chromosomes $\mathrm{X}$ and $\mathrm{Y}$ satellite arrays. Genome Res. 2014;24(4):697-707.

71. Kent WJ, et al. The human genome browser at UCSC. Genome Res. 2002;12(6):996-1006. 